\title{
Bayesian linear mixed models using Stan: A tutorial for psychologists, linguists, and cognitive scientists
}

\author{
Tanner Sorensen $^{\mathrm{a}, \otimes}$, Sven Hohenstein $^{\mathrm{b}} \&$ Shravan Vasishth $^{\mathrm{c}}$ \\ ${ }^{\text {a }}$ Signal Analysis and Interpretation Laboratory; University of Southern California \\ ${ }^{\mathrm{b}}$ Department of Psychology; University of Potsdam \\ ${ }^{\mathrm{c}}$ Department of Linguistics; University of Potsdam and CEREMADE; Université Paris-Dauphine
}

\begin{abstract}
With the arrival of the R packages $n l$ me and lme4, linear mixed models (LMMs) have come to be widely used in experimentally-driven areas like psychology, linguistics, and cognitive science. This tutorial provides a practical introduction to fitting LMMs in a Bayesian framework using the probabilistic programming language Stan. We choose Stan (rather than WinBUGS or JAGS) because it provides an elegant and scalable framework for fitting models in most of the standard applications of LMMs. We ease the reader into fitting increasingly complex LMMs, using a twocondition repeated measures self-paced reading study.
\end{abstract}

Keywords $\backsim$ Bayesian data analysis, linear mixed models. Tools $\backsim$ Stan, R.

Acting Editor $\square$ Denis Cousineau (Université d'Ottawa)

Reviewers

- Two anonymous reviewers

\tsorense@usc.edu

TS: 0000-0002-3111-9974; SH: 0000-0002-9708-1593; SV: 0000-0003-2027-1994

10.20982/tqmp.12.3.p175

\section{Introduction}

Linear mixed models, or hierarchical/multilevel linear models, have become the main workhorse of experimental research in psychology, linguistics, and cognitive science, where repeated measures designs are the norm. Within the programming environment $\mathrm{R}$ ( $\mathrm{R}$ Development Core Team, 2006), the nlme package (Pinheiro \& Bates, 2000) and its successor, lme 4 (Bates, Mächler, Bolker, \& Walker, 2015) have revolutionized the use of linear mixed models (LMMs) due to their simplicity and speed: one can fit fairly complicated models relatively quickly, often with a single line of code. A great advantage of LMMs over traditional approaches such as repeated measures ANOVA and paired t-tests is that there is no need to aggregate over subjects and items to compute two sets of F-scores (or several tscores) separately; a single model can take all sources of variance into account simultaneously. Furthermore, comparisons between conditions can easily be implemented in a single model through appropriate contrast coding.

Other important developments related to LMMs have been unfolding in computational statistics. Specifi- cally, probabilistic programming languages like WinBUGS (Lunn, Thomas, Best, \& Spiegelhalter, 2000), JAGS (Plummer, 2012) and Stan (Stan Development Team, 2014), among others, have made it possible to fit Bayesian LMMs quite easily. However, one prerequisite for using these programming languages is that some background statistical knowledge is needed before one can define the model. This difficulty is well-known; for example, Spiegelhalter, Abrams, and Myles (2004, p. 4) write: "Bayesian statistics has a (largely deserved) reputation for being mathematically challenging and difficult to put into practice...".

The purpose of this paper is to facilitate a first encounter with model specification in one of these programming languages, Stan. The tutorial is aimed primarily at psychologists, linguists, and cognitive scientists who have used lme 4 to fit models to their data, but who may have only a basic knowledge of the underlying LMM machinery. By "basic knowledge" we mean that they may not be able to answer some or all of these questions: what is a design matrix; what is contrast coding; what is a random effects variance-covariance matrix in a linear mixed model; what is the Cholesky decomposition? Our tutorial is not 
intended for statisticians or psychology researchers who could, for example, write their own Markov Chain Monte Carlo (MCMC) samplers in $\mathrm{R}$ or $\mathrm{C}++$ or the like; for them, the Stan manual is the optimal starting point. The present tutorial attempts to ease the beginner into their first steps towards fitting Bayesian linear mixed models. More detailed presentations about linear mixed models are available in several textbooks; references are provided at the end of this tutorial. For the complete newcomer to statistical methods, the articles by Vasishth and Nicenboim (2016) and Nicenboim and Vasishth (2016) should be read first, as they provide a grounds-up preparation for the present article.

We have chosen Stan as the programming language of choice (over JAGS and WinBUGS) because it is possible to fit arbitrarily complex models with Stan. For example, it is possible (if time consuming) to fit a model with 14 fixed effects predictors and two crossed random effects by subject and item, each involving a $14 \times 14$ variance-covariance matrix (Bates, Kliegl, Vasishth, \& Baayen, 2015); as far as we are aware, such models cannot be fit in JAGS or WinBUGS. ${ }^{1}$

In this tutorial, we take it as a given that the reader is interested in learning how to fit Bayesian linear mixed models. The tutorial is structured as follows. After a short introduction to Bayesian modeling, we begin by successively building up increasingly complex LMMs using the data-set reported by Gibson and Wu (2013), which has a simple two-condition design. At each step, we explain the structure of the model. The next section takes up inference for this two-condition design.

This paper was written using a literate programming tool, knitr (Xie, 2015); this integrates documentation for the accompanying code with the paper. The knitr file that generated this paper, as well as all the code and data used in this tutorial, can be downloaded from our website: https://www.ling.uni-potsdam.de/ vasishth/statistics/ BayesLMMs.html

In addition, the source code for the paper, all R code, and data are available on github at:

https://github.com/vasishth/BayesLMMTutorial

We start with the two-condition repeated measures data-set (Gibson \& Wu, 2013) as a concrete running example. This simple example serves as a starter kit for fitting commonly used LMMs in the Bayesian setting. We assume that the reader has the relevant software installed; specifically, the RStan interface to Stan in R. For detailed instructions, see https://github.com/stan-dev/rstan/wiki/RStan-Getting-Started

\footnotetext{
${ }^{1}$ Whether it makes sense in general to fit such a complex model is a different issue; see Gelman et al. (2013), and Bates, Kliegl, et al. (2015) for recent
discussion.

${ }^{1}$ Whether it makes sense in general to fit such a complex model is a different issue; see Gelman et al. (2013), and Bates, Kliegl, et al. (2015) for recent
discussion.
}

\section{Bayesian statistics}

Bayesian modeling has two major advantages over frequentist analysis with linear mixed models. First, information based on pre-existing knowledge can be incorporated into the analysis using different priors. Second, complex models with a large number of variance components can be fit. In the following, we will provide a short introduction to Bayesian statistics which highlights these two advantages of the Bayesian approach to data analysis.

The first advantage of the Bayesian approach is a consequence of Bayes' Theorem, the fundamental rule of Bayesian statistics. It can be seen as a way of understanding how the probability that a hypothesis is true is affected by new data. In mathematical notation, Bayes' Theorem states

$$
P(H \mid D)=\frac{P(D \mid H) P(H)}{P(D)}
$$

where $H$ is the hypothesis we are interested in and $D$ represents new data. Since $D$ is fixed for a given data-set, the theorem can be rephrased as

$$
P(H \mid D) \propto P(D \mid H) P(H)
$$

The posterior probability that the hypothesis is true given new data, $P(H \mid D)$, is proportional to the product of the likelihood of the new data given the hypothesis, $P(D \mid H)$, and the prior probability of the hypothesis, $P(H)$.

For the purposes of this paper, the goal of a Bayesian analysis is simply to derive the posterior distribution of each parameter of interest, given some data and prior knowledge about the distributions of the parameters. The following example illustrates how the posterior depends on the likelihood and prior. Before collecting data, a researcher has some hypothesis concerning the distribution of the response variable $X$ in an experiment. The reseacher expresses his or her belief in a prior distribution, say, a normal distribution with a mean value of $\mu=60$ and variance $\sigma^{2}=1000$ (solid density in left-hand panel of Figure 1). The large variance reflects the researcher's uncertainty concerning the true mean of the distribution. Alternatively, if the researcher were very certain that $\mu=60$, then he or she might choose the much lower variance $\sigma^{2}=100$ (solid density in right-hand panel of the righthand panel of Figure 1).

The researcher starts to collect data. In our example, there are $n=20$ values with a sample mean $\bar{x}=100$ and sample standard deviation $s=40$. The corresponding likelihood distribution is displayed in Figure 1 (dashed line). The resulting posterior distribution (dash-dot line) combines the prior and likelihood. Given the prior with the 
Figure 1 a Prior, likelihood, and posterior normal distributions. The likelihood is based on $n=20$ observations with sample mean $\mu=100$ and standard deviation $\sigma=40$. The prior (identical in both panels) has mean $\mu_{0}=60$ and variance $\sigma_{0}^{2}=1000$ (left-hand panel) or $\sigma_{0}^{2}=100$ (right-hand panel), respectively.

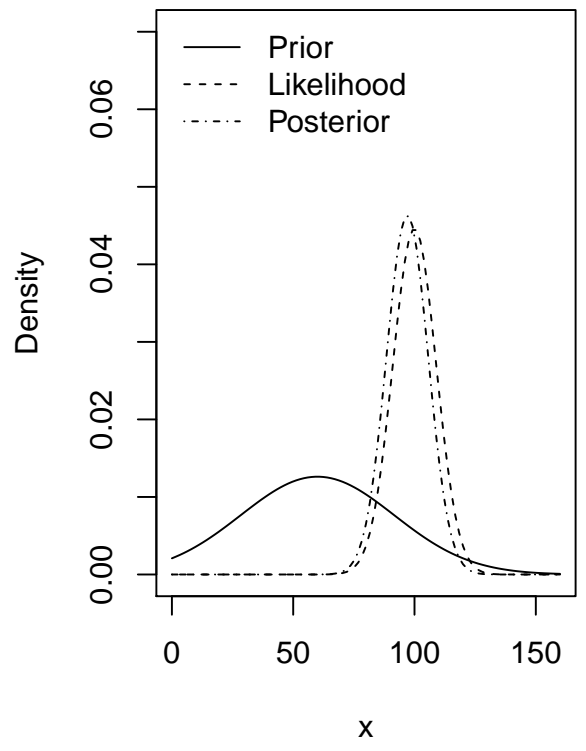

larger variance (left-hand panel), the posterior is largely influenced by the data. Given the prior with the smaller variance (right-hand panel), its influence on the posterior is much stronger, resulting in a smaller shift towards the data mean.

This toy example illustrates the central idea of Bayesian modeling. The prior reflects our knowledge of past results. In most cases, we will use so-called vague flat priors such that the posterior distribution is mainly affected by the data. The resulting posterior distribution allows for making inferences about model parameters.

The second advantage of Bayesian modeling concerns variance components (random effects). Fitting a large number of random effects in non-Bayesian settings requires a large amount of data. Often, the data-set is too small to reliably estimate variance component parameters (Bates, Kliegl, et al., 2015; Matuschek, Kliegl, Vasishth, Baayen, \& Bates, 2016). However, if a researcher is interested in differences between individual subjects or items (random intercepts and random slopes) or relationships between differences (correlations between variance components), Bayesian modeling can be used even if there is not enough data for inferential statistics. The resulting pos-

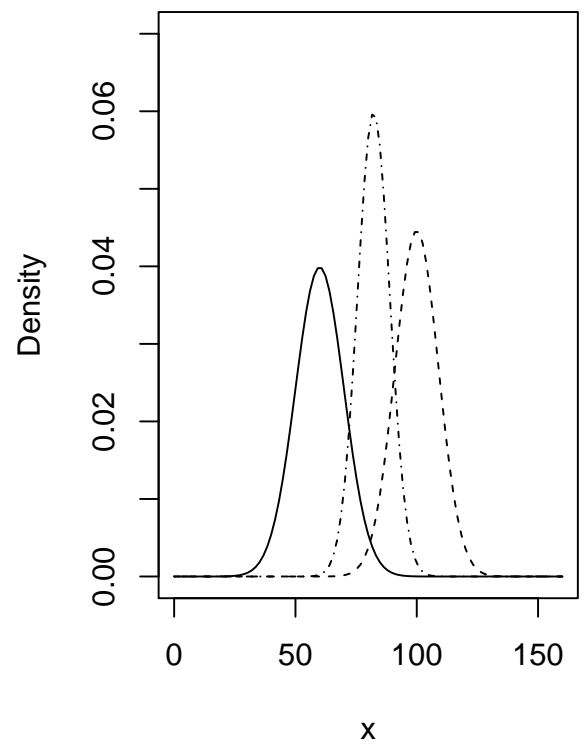

terior distributions might have high variance but they still allow for calculating probabilities of true parameter values of variance components. Note that we do not intend to criticize classical LMMs, but rather to highlight the possibilities of Bayesian modeling concerning random effects. For further explanation of the advantages this approach affords beyond the classical frequentist approach, the reader is directed to the rich literature relating to a comparison between Bayesian versus frequentist statistics (such as the provocatively titled paper by Lavine, 1999, and the highly accessible textbooks by mcelreath2016statistical mcelreath2016statistical and Kruschke, 2015).

\section{Example: A two-condition repeated measures design}

This section motivates the LMM with the self-paced reading data-set of Gibson and Wu (2013). We introduce the data-set, state our modeling goals here, and proceed to build up increasingly complex LMMs, starting with a fixed effects linear model before adding varying intercepts, adding varying slopes, and finally modeling the correlation between the varying intercepts and slopes (the "maximal model” of Barr, Levy, Scheepers, and Tily, 2013). We explain these new model parameters as we introduce them. 
Models of varying complexity such as these three can be generalized as described in Appendix . The result of our modeling is a probability model that expresses how the dependent variable, the reading time labeled $r t$, was generated in the experiment of Gibson and Wu (2013). The model allows us to derive the posterior probability distribution of the model parameters from a prior probability distribution and a likelihood function. Stan makes it easy to compute this posterior distribution for each model parameter of interest. The resulting posterior distribution reflects what we should believe about the value of that parameter, given the experimental data.

The scientific question. Subject and object relative clauses have been widely used in reading studies to investigate sentence comprehension processes. A subject relative is a sentence like The senator who interrogated the journalist resigned where a noun (senator) is modified by a relative clause (who interrogated the journalist), and the modified noun is the grammatical subject of the relative clause. In an object relative, the noun modified by the relative clause is the grammatical object of the relative clause (e.g., The senator who the journalist interrogated resigned). In both cases, the noun that is modified (senator) is called the head noun.

A typical finding for English is that subject relatives are easier to process than object relatives (Just \& Carpenter, 1992). Natural languages generally have relative clauses, and the subject relative advantage has until recently been considered to be true cross-linguistically. However, Chinese relative clauses apparently represent an interesting counter-example to this generalization; recent work by Hsiao and Gibson (2003) has suggested that in Chinese, object relatives are easier to process than subject relatives at a particular point in the sentence (the head noun of the relative clause). We now present an analysis of a subsequently published data-set (Gibson \& Wu, 2013) that evaluates this claim.

The data. The dependent variable of the experiment of Gibson and Wu (2013) was the reading time rt in milliseconds of the head noun of the relative clause. This was recorded in two conditions (subject relative and object relative), with 37 subjects and 15 items, presented in a standard Latin square design. There were originally 16 items, but one item was removed, resulting in $37 \times 15=555$ data points. However, eight data points from one subject (id 27) were missing. As a consequence, we have a total of $555-8=547$ data points. The first few lines from the data frame are shown in Table 1; “o” refers to object relative and "s" to subject relative.

\section{Fixed Effects Model}

We begin by making the working assumption that the dependent variable of reading time rt on the head noun is approximately log-normally distributed (Rouder, 2005). This assumes that the logarithm of $r t$ is approximately normally distributed. The logarithm of the reading times, $\log r t$, has some unknown grand mean $\beta_{0}$. The mean of the log-normal distribution of $r t$ is the sum of $\beta_{0}$ and an adjustment $\beta_{1}$ so whose magnitude depends on the categorical predictor so, which has the value -1 when $r t$ is from the subject relative condition, and 1 when $r t$ is from the object relative condition. One way to write the model in terms of the logarithm of the reading times is as follows:

$$
\log r \mathrm{t}_{i}=\beta_{0}+\beta_{1} \mathrm{so}_{i}+\varepsilon_{i}
$$

This is a fixed effects model. The index $i$ represents the $i$-th row in the data-frame (in this case, $i \in\{1, \ldots, 547\}$ ); the term $\varepsilon_{i}$ represents the error in the $i$-th row. With the above \pm 1 contrast coding, $\beta_{0}$ represents the grand mean of $\log r t$, regardless of relative clause type. It can be estimated by simply taking the grand mean of $\log r t$. The parameter $\beta_{1}$ is an adjustment to $\beta_{0}$ so that the mean of $\log r t$ is $\beta_{0}+1 \beta_{1}$ when $\log r t$ is from the object relative condition, and $\beta_{0}-1 \beta_{1}$ when log rt is from the subject relative condition. Notice that $2 \beta_{1}$ will be the difference in the means between the object and subject relative clause conditions. Together, $\beta_{0}$ and $\beta_{1}$ make up the part of the model which characterizes the effect of the experimental manipulation, relative clause type ( $\mathrm{s} \circ$ ), on the dependent variable rt. We call this a fixed effects model because we estimate the parameters $\beta_{0}$ and $\beta_{1}$, which do not vary from subject to subject or from item to item. In $\mathrm{R}$, this would correspond to fitting a simple linear model using the $1 \mathrm{~m}$ function, with so as predictor and $\log r t$ as dependent variable.

The error $\varepsilon_{i}$ is positive when $\log r t_{i}$ is greater than the expected value $\mu_{i}=\beta_{0}+\beta_{1} \mathrm{so}_{i}$ and negative when $\log r t_{i}$ is less than the expected value $\mu_{i}$. Thus, the error is the amount by which the expected value differs from actually observed value. We assume that the $\varepsilon_{i}$ are independently and identically distributed as a normal distribution with mean zero and unknown standard deviation $\sigma_{e}$. Stan parameterizes the normal distribution by the mean and standard deviation, and we follow that convention here by writing the distribution of $\varepsilon$ as $\mathcal{N}\left(0, \sigma_{e}\right)$. (This is different from the standard notation in statistics, where the normal distribution is defined in terms of mean and variance.) A consequence of the assumption that the errors are identically distributed is that the distribution of $\varepsilon$ should, at least approximately, have the same shape as the normal distribution. Independence implies that there should be no correlation between the errors-this is not the case in the data, since we have multiple measurements from each 
Table 1 a First six rows, and the last row, of the data-set of Gibson and Wu (2013), as they appear in the data frame.

\begin{tabular}{rrrlr}
\hline row & subj & item & so & rt \\
\hline 1 & 1 & 13 & 0 & 1561 \\
2 & 1 & 6 & s & 959 \\
3 & 1 & 5 & 0 & 582 \\
4 & 1 & 9 & 0 & 294 \\
5 & 1 & 14 & s & 438 \\
6 & 1 & 4 & s & 286 \\
$\vdots$ & $\vdots$ & $\vdots$ & $\vdots$ & \\
547 & 9 & 11 & 0 & 350 \\
\hline
\end{tabular}

subject and multiple measurements from each item. This introduces correlation between errors.

Setting up the data. We now fit the fixed effects model. For the following discussion, refer to the code in Listings 1 ( $\mathrm{R}$ code) and 2 (Stan code). First, we read the Gibson and $\mathrm{Wu}$ (2013) data into a data frame rDat in R, and then subset the critical region (Listing 1, lines 2 and 4). Next, we create a data list stanDat for Stan, which contains the data (line 13). Stan requires the data to be of type list; this is different from the $1 \mathrm{~m}$ and 1 mer functions, which assume that the data are of type data-frame.

Defining the model. The next step is to write the Stan model in a text file with extension . stan. A Stan model consists of several blocks. A block is a set of statements surrounded by brackets and preceded by the block name. We open up a file fixEf. stan in a text editor and write down the first block, the data block, which contains the declaration of the variables in the data object stanDat (Listing 2, lines 1-5). The strings real and int specify the data type for each variable. A real variable is a real number, and an int variable is an integer. For instance, $\mathrm{N}$ is the integer number of data points. The variables so and rt are arrays of length $\mathrm{N}$ whose entries are real. We constrain a variable to take only a subset of the values allowed by its type (e.g., int or real) by specifying in brackets lower and upper bounds (e.g. <lower $=-1$, upper $=1>$ ). The variables in the data block, $\mathrm{N}, \mathrm{rt}$, and so, correspond to the values of the list stanDat in R. The list stanDat must match the variables of the data block in case, but the order of variable declarations in the data block does not necessarily have to match the order of values in the list stanDat.

Next, we turn to the parameters block, where the parameters are defined (Listing 2, lines 6-9). These are the model parameters, for which posterior distributions are of interest. The fixed effects model has three parameters: the fixed intercept $\beta_{0}$, the fixed slope $\beta_{1}$, and the standard deviation $\sigma_{e}$ of the error. We store the fixed effects $\beta_{0}$ and $\beta_{1}$ in a vector, which contains variables of type real. Although we called our parameters $\beta_{0}$ and $\beta_{1}$ in the fixed effects model, in Stan, these are contained in the vector beta with indices 1 and 2 . Thus, $\beta_{0}$ is in beta [1] and $\beta_{1}$ in beta [2]. The third parameter, the standard deviation $\sigma_{e}$ of the error (sigma_e), is also defined here, and is constrained to have lower bound zero (Listing 2, line 8).

Finally, the model block specifies the prior distribution and the likelihood (Listing 2, lines 10-16). To understand the Stan syntax, compare the Stan code above to the specification of the fixed effects model. The Stan code literally writes out this model. The block begins with a local variable declaration for mu, which is the mean of rt conditional on whether so is -1 for the subject relative condition or 1 for the object relative condition.

The for-loop assigns to mu the mean for the log-normal distribution of $r t[i]$, conditional on the value of the predictor so[i] for relative clause type. The statement rt [i] lognormal (mu, sigma_e) in a forloop means that the logarithm of each value in the vector $r t$ is normally distributed with mean mu and standard deviation sigma_e. ${ }^{2}$

The prior distributions on the parameters beta and sigma_e would ordinarily be declared in the model block. If we don't declare any prior, it is assumed that they have a uniform prior distribution. Note that the distribution of sigma_e is truncated at zero because sigma_e is constrained to be positive (see the declaration real<lower=0> sigma_e; in the parameters block). This means that the error has a uniform prior with lower bound zero. ${ }^{3}$

\footnotetext{
${ }^{2}$ One could have equally well log-transformed the reading time and assumed a normal distribution instead of the lognormal.

${ }^{3}$ This is an example of an improper prior, which is not a probability distribution. Although all the improper priors used in this tutorial produce posteriors which are probability distributions, this is not true in general, and care should be taken in using improper priors (Gelman, 2006). In the present case, a Cauchy prior truncated to have a lower bound of 0 could alternatively be defined for the standard deviation. For example code using such a prior, see the KBStan vignette in the ReP sychLing package (Baayen, Bates, Kliegl, \& Vasishth, 2015).
} 
Listing 1 - R code for the fixed effects model.

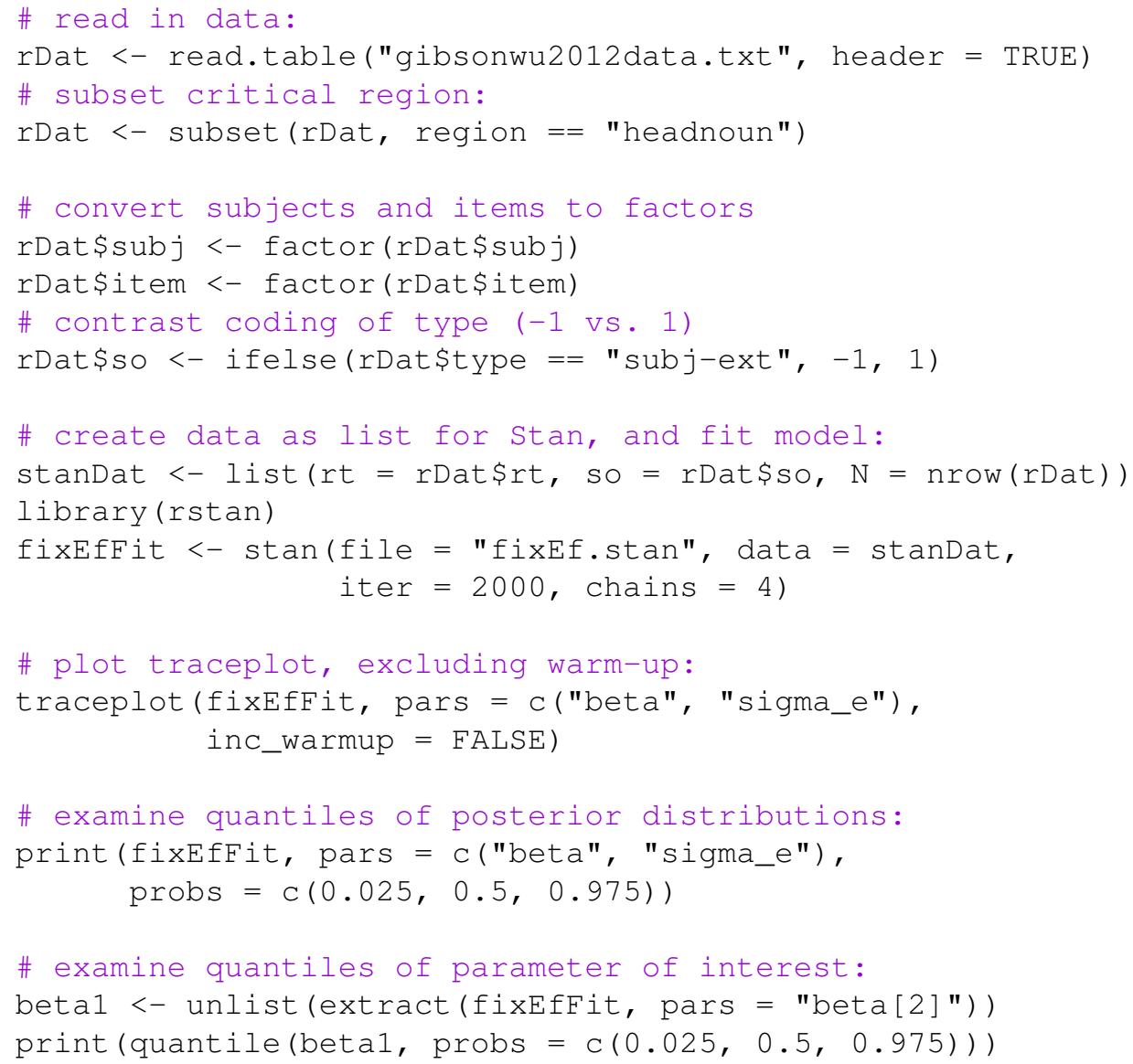

Running the model. We save the file fixEf. stan which contains the Stan code and fit the model in R with the function st an from the package rstan (Listing 1, lines 15-16). This call to the function stan will compile a $\mathrm{C}++$ program which produces samples from the joint posterior distribution of the fixed intercept $\beta_{0}$, the fixed slope $\beta_{1}$, and the standard deviation $\sigma_{e}$ of the error.

The function generates four chains of samples. A Markov chain is a stochastic process in which random values are sequentially generated. Each sample depends on the previous one. Different chains are independent of each other such that running a Stan model with four chains is equivalent to running four (identically specified) Stan models with one chain each. For the model used here, each of the four chains contains 2000 samples of each parameter.

Samples 1 to 1000 are part of the warmup, where the chains settle into the posterior distribution. We analyze samples 1001 to 2000 . The result is saved to an object
fixEfFit of class stanFit.

The warmup samples, also known as the burn-in period, are intended to allow the MCMC sampling process to converge to the posterior distribution. Once a chain has converged, the samples remain quite stable. ${ }^{4}$ Before the MCMC sampling process, the number of interations necessary for convergence is unknown. Therefore, all warmup samples are discarded. This is necessary since the initial values of the parameters might have low posterior probability and might therefore bias the result.

Besides the number of samples, we specified sampling in four different chains. Each chain is independent from the others and starts with different random initial values. Running multiple chains has two advantages over a single chain. First, the independent chains are helpful for diagnostics. If all chains have converged to the same region of the parameter space, it is more likely that they converged to the posterior distribution. Second, running multiple chains allows for parallel simulations on multiple

\footnotetext{
${ }^{4}$ See, Gelman et al., 2013 for a precise discussion of convergence.
} 
Listing 2 - Stan code for the fixed effects model.

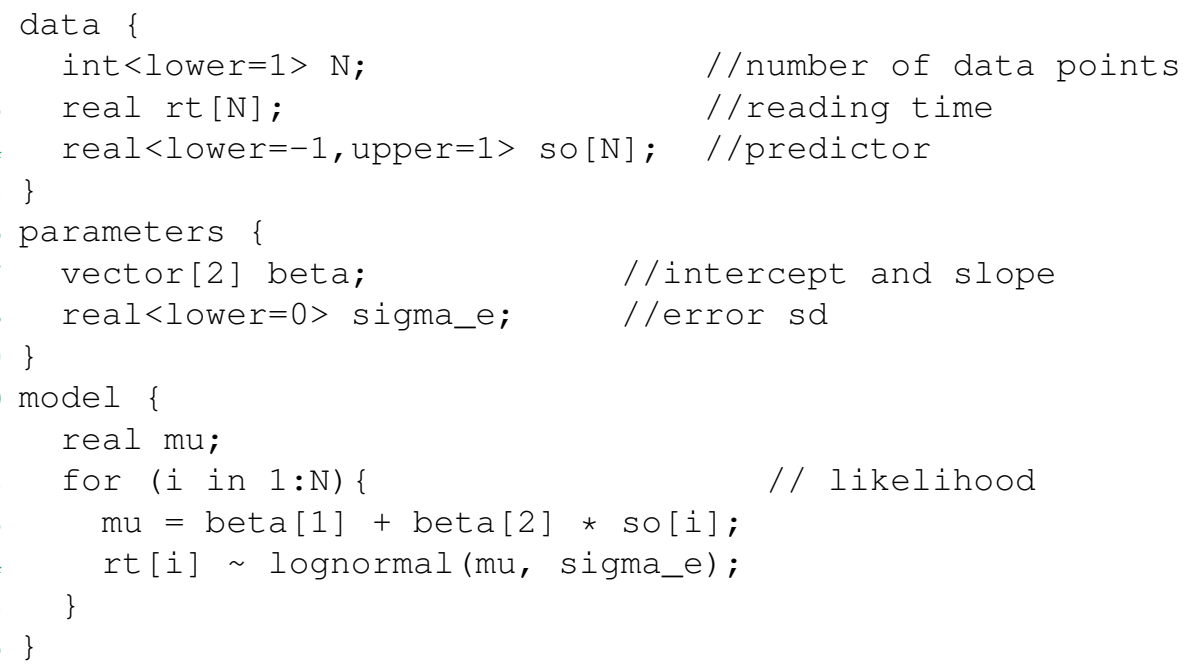

cores.

Evaluating model convergence. The number of iterations necessary for convergence to the posterior distribution depends on the number of parameters. The probability of reaching convergence increases with the number of iterations. Hence, we generally recommend using a large number of iterations although the process might converge after a smaller number of iterations. In the examples in the present paper, we use 1000 iterations for warmup and another 1000 iterations for analyzing the posterior distribution. For more complex models, more iterations might be necessary before the MCMC sampling process converges to the posterior distribution. Although there are ways to determine how long the simulation needs to be run and the number of warmup iterations given the type of posterior distribution (Raftery \& Lewis, 1992), we illustrate below practical convergence diagnostics for the evaluation of convergence in the samples.

The first step after running the function stan should be to look at the trace plot of each chain after warmup, using the command shown in Listing 1, lines 13 and 14 (function traceplot). We choose the parameters $\beta_{i}$ and $\sigma_{e}($ pars $=$ c ("beta", "sigma_e")) and omit the warmup samples (inc_warmup = FALSE). A trace plot has the chains plotted against the sample number. In Figure 2, we see three different chains plotted against sample number going from 1001 to 2000. If the trace plot looks like a "fat, hairy caterpillar" (Lunn, Jackson, Spiegelhalter, Best, \& Thomas, 2012) which does not bend, this suggests that the chains have converged to the posterior distribution.

The second diagnostic which we use to assess whether the chains have converged to the posterior distribution is the statistic Rhat. Each parameter has the Rhat statistic associated with it (Gelman \& Rubin, 1992); this is essentially the ratio of between-chain variance to within-chain variance (analogous to ANOVA). The Rhat statistic should be approximately $1 \pm 0.1$ if the chain has converged. This is shown in the rightmost column of the model summary, printed in Table 2. The information can be otained with print (fixEfFit), where fixEfFit is the object of type stan. model returned by the function stan. For example, see Listing 1, lines 23-24.

Having satisfied ourselves that the chains have converged, next we turn to examine this posterior distribution. If there is an indication that convergence has not happened, then, assuming that the model has no errors in it, increasing the number of samples usually resolves the issue.

Summarizing the result. The result of fitting the fixed effects model is the joint posterior probability distribution of the parameters $\beta_{0}, \beta_{1}$, and $\sigma_{e}$. The distribution is joint because each of the 4000 ( 4 chains $\times$ 1000 post-warmup iterations) posterior samples which the call to stan generates is a vector $\theta=\left(\beta_{0}, \beta_{1}, \sigma_{e}\right)^{\top}$ of three model parameters. Thus, the object $f i x E f F i t$ contains 4000 parameter vectors $\theta$ which occupy a three dimensional space. Already in three dimensions, the posterior distribution becomes difficult to view in one graph. Figure 3 displays the joint posterior probability distribution of the elements of $\theta$ by projecting it down onto planes. In each of the three planes (lower triangular scattergrams) we see how one parameter varies with respect to the other. In the diagonal histograms, we visualize the marginal probability distribution of each parameter separately from the other parameters. 
Figure 2 - Trace plots of the fixed intercept $\beta_{0}$ (beta [1] ), the fixed slope $\beta_{1}$ (beta [2] ), and the standard deviation $\sigma_{e}$ (sigma_e) of the error for the fixed effects model. Different colours denote different chains.

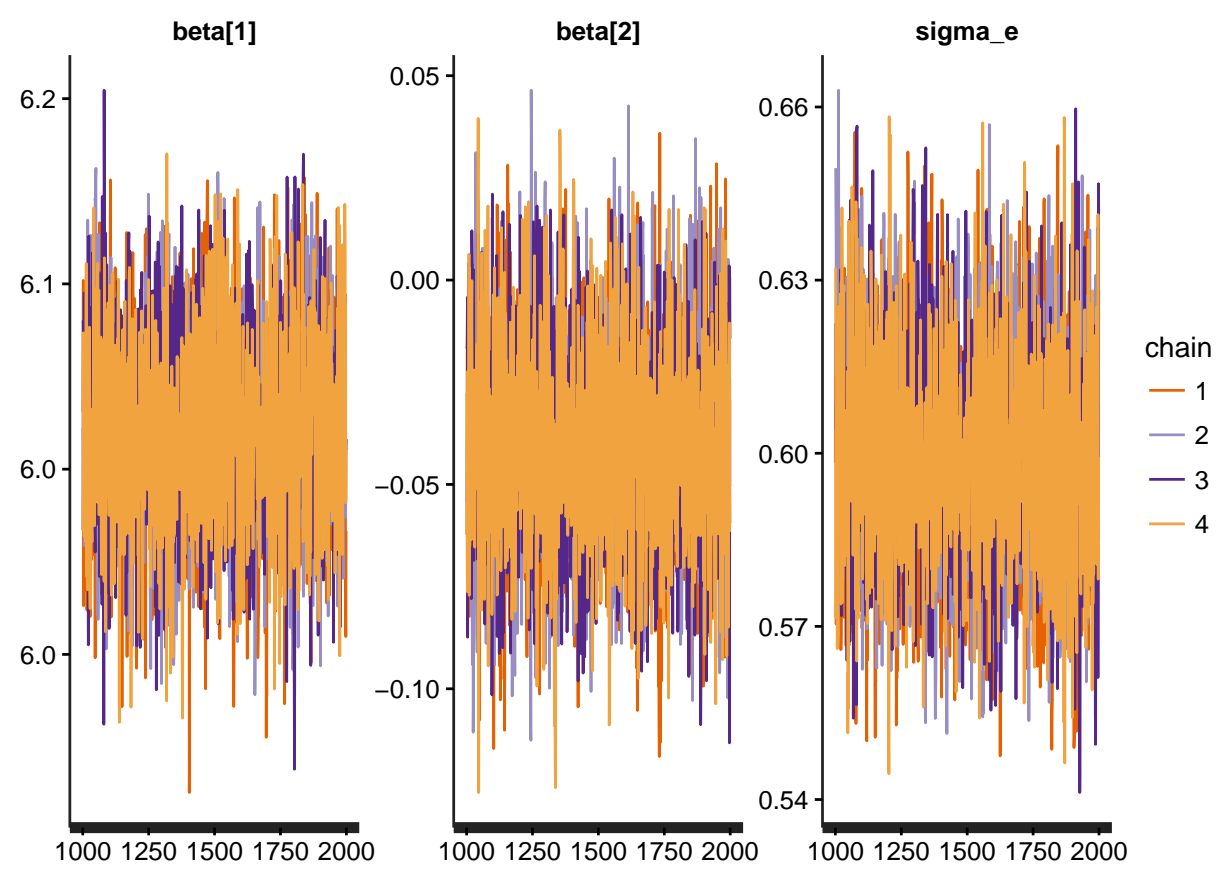

Table 2 - Credible intervals and R-hat statistic in the Gibson and Wu data.

\begin{tabular}{crrrr}
\hline parameter & mean & $2.5 \%$ & $97.5 \%$ & $\hat{R}$ \\
\hline$\hat{\beta}_{0}$ & 6.06 & 6.01 & 6.12 & 1 \\
$\hat{\beta}_{1}$ & -0.04 & -0.09 & 0.02 & 1 \\
$\hat{\sigma}_{e}$ & 0.60 & 0.56 & 0.64 & 1 \\
\hline
\end{tabular}

Of immediate interest is the marginal distribution of the slope $\beta_{1}$. Figure 3 suggests that most of the posterior probability density of $\beta_{1}$ is located below zero. One quantitative way to assess the posterior probability distribution is to examine its quantiles; see Table 2. Here, it is useful to define the concept of the credible interval. The $(1-\alpha) \%$ credible interval contains $(1-\alpha) \%$ of the posterior probability density. Unlike the $(1-\alpha) \%$ confidence interval from the frequentist setting, the $(1-\alpha) \%$ credible interval represents the range within which we are $(1-\alpha) \%$ certain that the true value of the parameter lies, given the prior and the data (see Morey, Hoekstra, Rouder, Lee, and Wagenmakers, 2015 for further discussion on confidence intervals vs credible intervals). A common convention is to use the interval ranging from the 2.5th to 97.5th percentiles. We follow this convention to obtain $95 \%$ credible intervals in Table 2. Lines 27-28 of Listing 1 illustrate how these quantiles of the posterior distribution of $\beta_{1}$ (beta [2] ) can be computed.

The sample distribution of $\beta_{1}$ indicates that approximately $94 \%$ of the posterior probability density is below zero, suggesting that there is some evidence that object relatives are easier to process than subject relatives in Chinese, given the Gibson and Wu data. However, since the 95\% credible interval includes zero, we may be reluctant to draw this conclusion. We will say more about the evaluation of research hypotheses further on, but it is important to note here that the fixed effects model presented above is in any case not appropriate for the present data. The independence assumption is violated for the errors because we have repeated measures from each subject and from each item. Linear mixed models extend the linear model to solve precisely this problem. 
Figure 3 - Samples and level curves of the bivariate joint posterior probability distribution of each element of $\theta$ with each other element (lower triangular) and marginal posterior probability distribution of each element of $\theta$ separately (diagonal). All parameters are on the log scale, but note the difference in length scale between $\beta_{1}$ on the one hand and $\beta_{0}$ and $\sigma_{e}$ on the other.
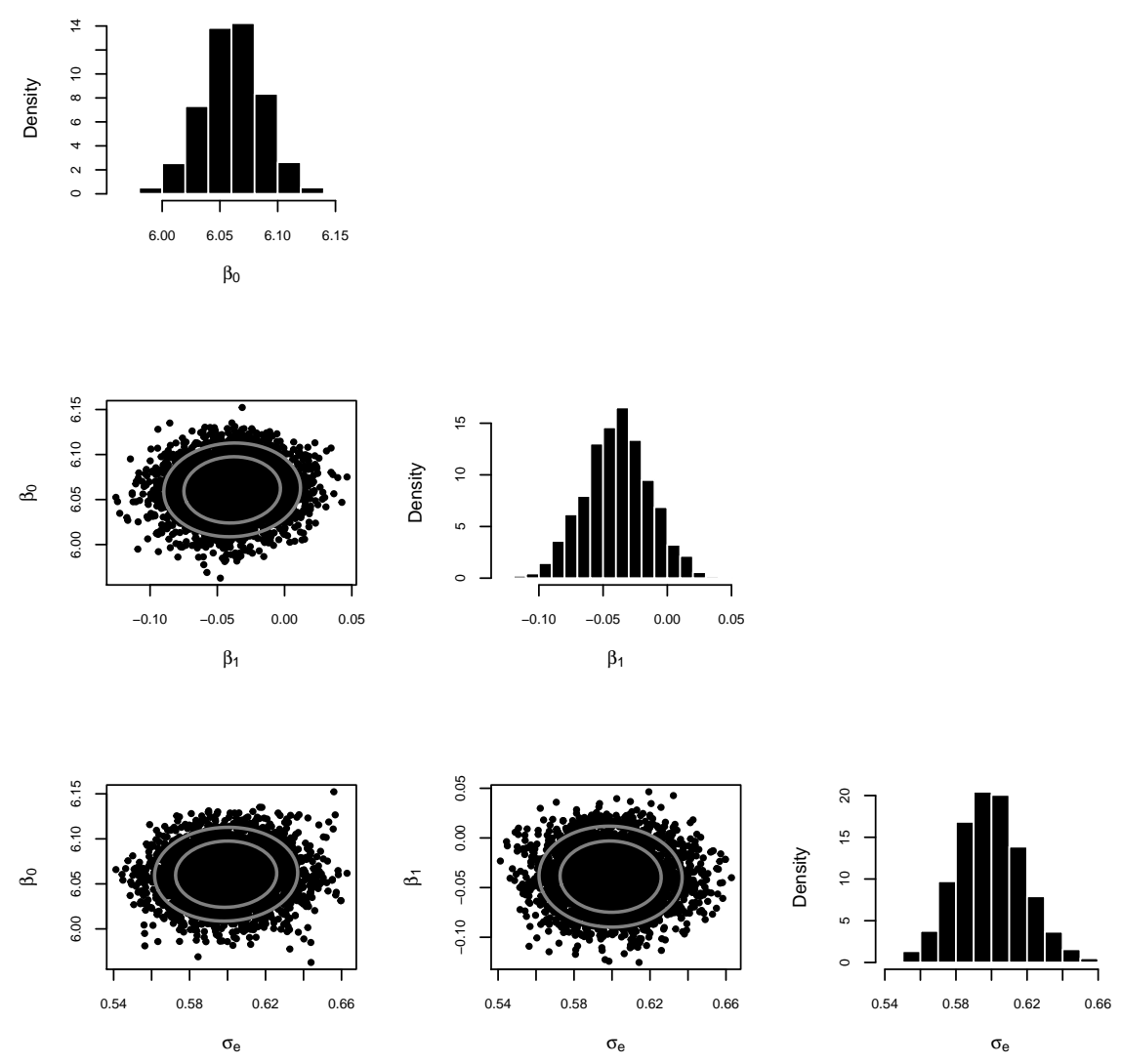

\section{Varying Intercepts Mixed Effects Model}

The fixed effects model is inappropriate for the Gibson and Wu data because it does not take into account the fact that we have multiple measurements for each subject and item. As mentioned above, these multiple measurements lead to a violation of the independence of errors assumption. Moreover, the fixed effects coefficients $\beta_{0}$ and $\beta_{1}$ represent means over all subjects and items, ignoring the fact that some subjects will be faster and some slower than average; similarly, some items will be read faster than average, and some slower.

In linear mixed models, we take this by-subject and byitem variability into account by adding adjustment terms $u_{0 j}$ and $w_{0 k}$, which adjust $\beta_{0}$ for subject $j$ and item $k$. This partially decomposes $\varepsilon_{i}$ into a sum of the terms $u_{0 j}$ and $w_{0 k}$, which are adjustments to the intercept $\beta_{0}$ for the subject $j$ and item $k$ associated with $r t_{i}$. If subject $j$ is slower than the average of all the subjects, $u_{j}$ would be some positive number, and if item $k$ is read faster than the average reading time of all the items, then $w_{k}$ would be some negative number. Each subject $j$ has their own adjustment $u_{0 j}$, and each item its own $w_{0 k}$. These adjustments $u_{0 j}$ and $w_{0 k}$ are called random intercepts by Pinheiro and Bates (2000) and varying intercepts by Gelman and Hill (2007), and by adjusting $\beta_{0}$ by these we account for the variability by speaker and by item.

We assume that these adjustments are normally distributed around zero with unknown standard deviation: $u_{0} \sim \mathcal{N}\left(0, \sigma_{u}\right)$ and $w_{0} \sim \mathcal{N}\left(0, \sigma_{w}\right)$. We now have three sources of variance in this model: the standard deviation of the errors $\sigma_{e}$, the standard deviation of the by-subject 
random intercepts $\sigma_{u}$, and the standard deviation of the by-item varying intercepts $\sigma_{w}$. We will refer to these as variance components.

We now express the logarithm of reading time, which was produced by subjects $j \in\{1, \ldots, 37\}$ reading items $k \in\{1, \ldots, 15\}$, in conditions $i \in\{1,2\}$ (1 refers to subject relatives, 2 to object relatives), as the following sum. Notice that we are now using a slightly different way to describe the model, compared to the fixed effects model. We are using indices for subject, item, and condition to identify unique rows. Also, instead of writing $\beta_{1} \mathrm{so}_{i}$, we index $\beta_{1}$ by the condition $i$. This follows the notation used in the textbook on linear mixed models, written by the authors of nlme (Pinheiro \& Bates, 2000), the precursor to lme 4.

$$
\log r t_{i j k}=\beta_{0}+\underbrace{\beta_{1 i}}_{\beta_{1} \mathrm{SO}_{i}}+u_{0 j}+w_{0 k}+\varepsilon_{i j k}
$$

This is an LMM, and more specifically a varying intercepts model. The coefficient $\beta_{1 i}$ is the one of primary interest; it will have some mean value $-\beta_{1}$ for subject relatives and $\beta_{1}$ for object relatives due to the contrast coding. So, if our posterior mean for $\beta_{1}$ is negative, this would suggest that object relatives are read faster than subject relatives.

We fit the varying intercepts model in Stan in much the same way as the fixed effects model. For the following discussion, consult Listing 3 for the $\mathrm{R}$ code used to run the model, and Listing 4 for the Stan code.

Setting up the data. The data which we prepare for passing on to the function stan now includes subject and item information (Listing 3, lines 2-8). The data block in the Stan code accordingly includes the number $\mathrm{J}, \mathrm{K}$ of subjects and items, respectively, as well as subject and item identifiers subj and item (Listing 4, lines 5-8).

Defining the model. The random intercepts model, shown in Listing 4 , still has the fixed intercept $\beta_{0}$, the fixed slope $\beta_{1}$, and the standard deviation $\sigma_{e}$ of the error, and we specify these in the same way as we did for the fixed effects model. In addition, the varying intercepts model has by-subject varying intercepts $u_{0 j}$ for $j \in\{1, \ldots, J\}$ and by-item varying intercepts $w_{0 k}$ for $k \in\{1, \ldots, K\}$. The standard deviation of $u_{0}$ is $\sigma_{u}$ and the standard deviation of $w_{0}$ is $\sigma_{w}$. We again constrain the standard deviations to be positive.

The model block places normal distribution priors on the varying intercepts $u_{0}$ and $w_{0}$. We implicitly place uniform priors on sigma_u, sigma_w, and sigma_e by omitting them from the model block. As pointed out earlier for sigma_e, these prior distributions have lower bound zero because of the constraint $<$ lower $=0>$ in the variable declarations.

The statement about how each row in the data is gen- erated is shown in Listing 4, lines 26-29; here, both the fixed effects and the varying intercepts for subjects and items determine the expected value mu. The vector $u$ has varying intercepts for subjects. Likewise, the vector $\mathrm{w}$ has varying intercepts for items. The for-loop in lines 2629 now adds u [subj [i] ] + w [item [i] ] to the mean beta [1] of the distribution of rt [i] . These are subjectand item-specific adjustments to the fixed-effects intercept beta [1]. The term $u[s u b j[i]]$ is the identifier of the subject for row $i$ in the data-frame; thus, if $i=1$, then subj[1] = 1, and item [1] = 13 (see Table 1).

Running the model. In $\mathrm{R}$, we pass the list stanDat of data to stan, which compiles a $\mathrm{C}++$ program to sample from the posterior distribution of the random intercepts model. Stan samples from the posterior distribution of the model parameters, including the varying intercepts $u_{0 j}$ and $w_{0 k}$ for each subject $j \in\{1, \ldots, J\}$ and item $k \in\{1, \ldots, K\}$.

It may be helpful to rewrite the model in mathematical form following the Stan syntax (Gelman and Hill, 2007 use a similar notation); the Stan statements are slightly different from the way that we expressed the random intercepts model. Defining $i$ as the row number in the data frame, i.e., $i \in\{1, \ldots, 547\}$, we can write:

$$
\begin{aligned}
& \text { Likelihood : } \\
& \mu_{i}=\beta_{0}+u_{[\operatorname{subj}[i]]}+w_{[i t e m[i]]}+\beta_{1} \cdot \mathrm{so}_{i} \\
& r t_{i} \sim \operatorname{LogNormal}\left(\mu_{i}, \sigma_{e}\right) \\
& \text { Priors : } \\
& u \sim \operatorname{Normal}\left(0, \sigma_{u}\right) \quad w \sim \operatorname{Normal}\left(0, \sigma_{w}\right) \\
& \sigma_{e}, \sigma_{u}, \sigma_{w} \sim \operatorname{Uniform}(0, \infty) \\
& \beta \sim \operatorname{Uniform}(-\infty, \infty)
\end{aligned}
$$

Here, notice that the $i$-th row in the statement for $\mu$ identifies the subject identifier $(j$ ) ranging from 1 to 37 , and the item identifier ( $k$ ) ranging from 1 to 15.

Summarizing the results. The posterior distributions of each of the parameters is summarized in Table 3. The $\hat{R}$ values suggest that model has converged because they equal one. Note also that compared to Model 1, the estimate of $\sigma_{e}$ is smaller; this is because the other two variance components are now being estimated as well. Note that the $95 \%$ credible interval for the estimate $\hat{\beta}_{1}$ includes zero; thus, there is some evidence that object relatives are easier than subject relatives, but we cannot exclude the possibility that there is no difference in the reading times between the two relative clause types.

\section{Varying Intercepts, Varying Slopes Mixed Effects Model}

The varying intercepts model accounted for having multiple measurements from each subject and item by introduc- 
Listing 3 = R code for running the random intercepts model, the varying intercepts model. Note that lines 1-10 and 14 of Listing 1 must be run first.

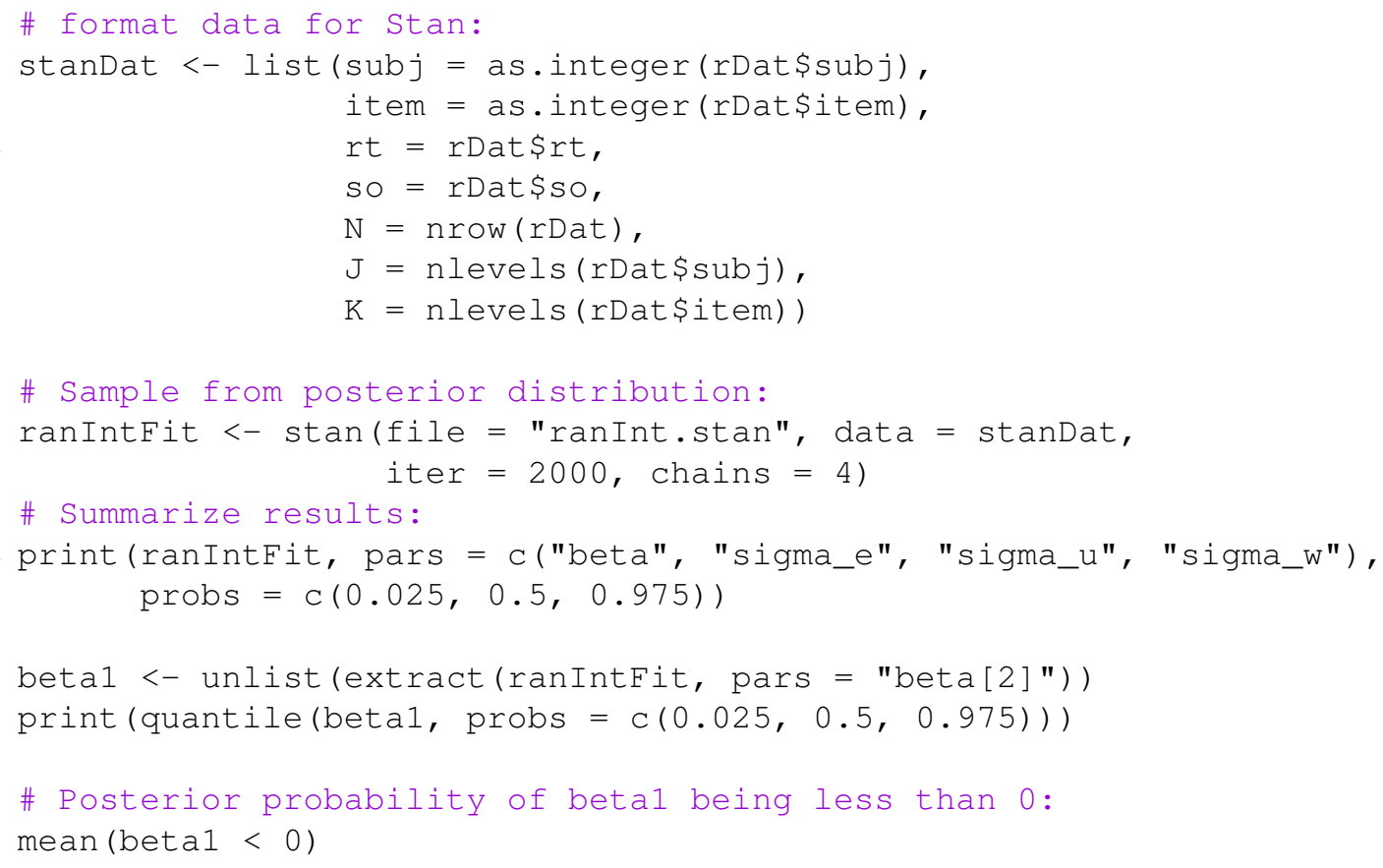

Table 3 - The quantiles and the $\hat{R}$ statistic in the Gibson and Wu data, the varying intercepts model.

\begin{tabular}{crrrr}
\hline parameter & mean & $2.5 \%$ & $97.5 \%$ & $\hat{R}$ \\
\hline$\hat{\beta}_{0}$ & 6.06 & 5.92 & 6.20 & 1 \\
$\hat{\beta}_{1}$ & -0.04 & -0.08 & 0.01 & 1 \\
$\hat{\sigma}_{e}$ & 0.52 & 0.49 & 0.56 & 1 \\
$\hat{\sigma}_{u}$ & 0.26 & 0.19 & 0.34 & 1 \\
$\hat{\sigma}_{w}$ & 0.20 & 0.12 & 0.33 & 1 \\
\hline
\end{tabular}

ing random intercepts by subject and by item. This reflects that some subjects will be faster and some slower than average, and that some items will be read faster than average, and some slower. Consider now that not only does reading speed differ by subject and by item, but also the slowdown in the object relative condition may differ in magnitude by subject and item. This amounts to a different effect size for so by subject and item. Although such individual-level variability was not of interest in the original paper by Gibson and $\mathrm{Wu}$, it could be of theoretical interest (see, for example, Kliegl, Wei, Dambacher, Yan, and Zhou, 2010). Furthermore, as Barr et al. (2013) point out, it is in principle desirable to include a fixed effect factor in the random effects as a varying slope if the experiment design is such that subjects see both levels of the factor (cf. Bates, Kliegl, et al., 2015; Matuschek et al., 2016; Baayen, Vasishth, Bates, \& Kliegl, 2016).
Adding varying slopes. In order to express this structure in the LMM, we must introduce varying slopes. The first change is to let the size of the effect for so vary by subject and by item. The goal here is to express that some subjects exhibit greater slowdowns in the object relative condition than others. We let effect size vary by subject and by item by including in the model by-subject and byitem varying slopes which adjust the fixed slope $\beta_{1}$ in the same way that the by-subject and by-item varying intercepts adjust the fixed intercept $\beta_{0}$. This adjustment of the slope by subject and by item is expressed by adjusting $\beta_{1}$ by adding two terms $u_{1 j}$ and $w_{1 k}$. These are random or varying slopes, and by adding them we account for how the effect of relative clause type varies by subject $j$ and by item $k$. We now express the logarithm of reading time, which was produced by subject $j$ reading item $k$, as the following sum. The subscript $i$ indexes the conditions. 
Listing 4 a Stan code for running the random intercepts model, the varying intercepts model.

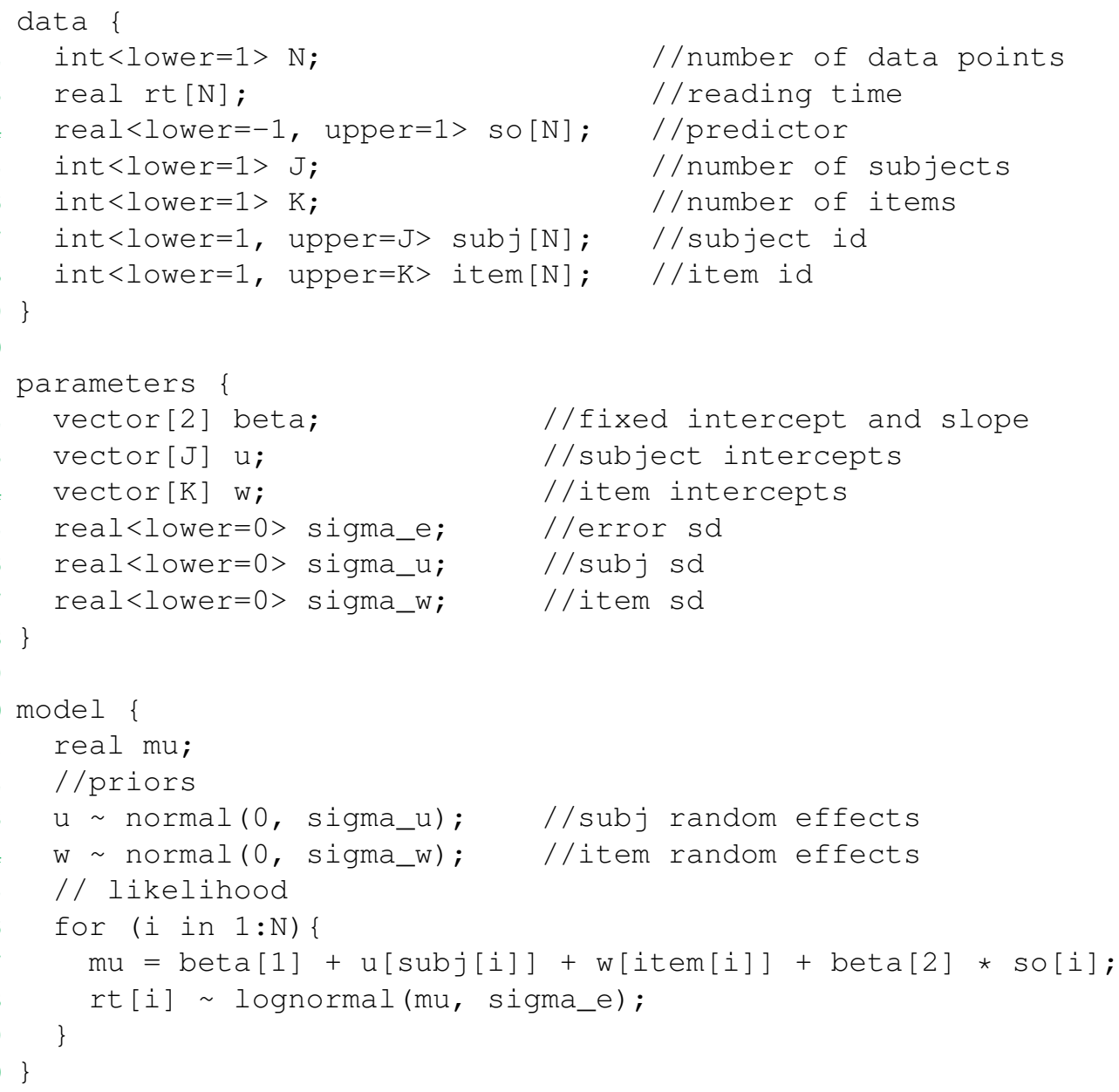

$$
\log r t_{i j k}=\underbrace{\beta_{0}+u_{0 j}+w_{0 k}}_{\text {varying intercepts }}+\underbrace{\beta_{1 i}+u_{1 i j}+w_{1 i k}}_{\text {varying slopes }}+\varepsilon_{i j k}
$$

This is a varying intercepts, varying slopes model.

Setting up the data. Listing 5 contains the R code for fitting the varying intercepts, varying slopes model. The data which we pass to the function stan is the same as for the varying intercepts model. This contains subject and item information (Listing 3, lines 2-8).

Defining the model. Listing 6 contains the Stan code for the varying intercepts, varying slopes model. The data block is the same as in the varying intercepts model, but the parameters block contains several new parameters. This time we have the vector sigma_u, which contains the standard deviations $\left(\sigma_{u 0}, \sigma_{u 1}\right)^{\top}$ of the by-subject random intercepts and slopes. The by-subject random intercepts are in the first row of the $2 \times \mathrm{J}$ matrix $u$, and the by- subject random slopes are in the second row of $u$. Similarly, the vector sigma_w contains the standard deviations $\left(\sigma_{w 0}, \sigma_{w 1}\right)^{\top}$ of the by-item random intercepts and slopes. The by-item random intercepts are in the first row of the $2 \times \mathrm{K}$ matrix $\mathrm{w}$, and the by-item random slopes are in the second row of $\mathrm{w}$.

In the model block, we place priors on the parameters declared in the parameters block (Listing 6, lines 23-26), and define how these parameters generate logrt (Listing 6, lines 28-32). The statement $u[1] \sim$ normal (0, sigma_u[1]); specifies a normal prior for the by-subject random intercepts in the first row of $u$, and the statement $u[2] \sim$ normal (0,sigma_u[2]); does the same for the bysubject random slopes in the second row of $u$. The same goes for the by-item random intercepts and slopes. Thus, there is a prior normal distribution for each of the random effects. These distributions are centered on zero and have different standard deviations. 
Listing 5 - R code for running the varying intercepts, varying slopes model. Note that lines 1-10 and 14 of Listing 1 and lines 2-8 of Listing 3 must be run first.

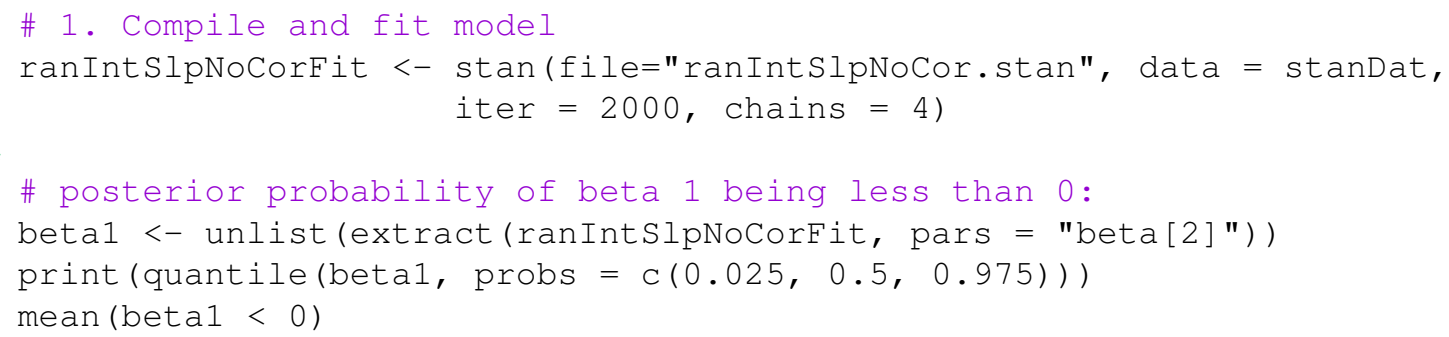

Running the model. We can now fit the varying intercepts, varying slopes model in R (see Listing 5). We see in the model summary of Table 4 , obtained as before using print (ranIntSlpNoCorFit), that the model has converged, and that the credible interval of the parameter of interest, $\beta_{1}$, still includes zero. In fact, the posterior probability of the parameter being less than zero is now $90 \%$.

\section{Correlated Varying Intercepts, Varying Slopes Mixed Effects Model}

Consider now that subjects who are faster than average (i.e., who have a negative varying intercept) may exhibit greater slowdowns when they read object relatives compared to subject relatives. Similarly, it is in principle possible that items which are read faster (i.e., which have a large negative varying intercept) may show a greater slowdown in the object relative condition than in the subject relative condition. The opposite situation could also hold: faster subjects may show smaller SR-OR effects, or items read faster may show smaller SR-OR effects. This suggests the possibility of correlations between random intercepts and random slopes.

In order to express this structure in the LMM, we must model correlation between the varying intercepts and varying slopes. The model equation, repeated below, is the same as before.

$$
\log r t_{i j k}=\underbrace{\beta_{0}+u_{0 j}+w_{0 k}}_{\text {varying intercepts }}+\underbrace{\beta_{1}+u_{1 i j}+w_{1 i k}}_{\text {varying slopes }}+\varepsilon_{i j k}
$$

Introducing correlation between the varying intercepts and varying slopes makes this a correlated varying intercepts, varying slopes model.

Defining a variance-covariance matrix for the random effects. Modeling the correlation between varying intercepts and slopes means defining a covariance relationship between by-subject varying intercepts and slopes, and between by-items varying intercepts and slopes. This amounts to adding an assumption that the by-subject slopes $u_{1}$ could in principle have some correlation with the by-subject intercepts $u_{0}$; and by-item slopes $w_{1}$ with by-item intercept $w_{0}$. We explain this in detail below.

Let us assume that the adjustments $u_{0}$ and $u_{1}$ are normally distributed with mean zero and some variances $\sigma_{u 0}^{2}$ and $\sigma_{u 1}^{2}$, respectively; also assume that $u_{0}$ and $u_{1}$ have correlation $\rho_{u}$. It is standard to express this situation by defining a variance-covariance matrix $\Sigma_{u}$, sometimes called simply a variance matrix. This matrix has the variances of $u_{0}$ and $u_{1}$ respectively along the diagonal, and the covariances on the off-diagonal. The covariance $\operatorname{Cov}(X, Y)$ between two variables $X$ and $Y$ is defined as the product of their correlation $\rho$ and their standard deviations $\sigma_{X}$ and $\sigma_{Y}: \operatorname{Cov}(X, Y)=\rho \sigma_{X} \sigma_{Y}$.

$$
\Sigma_{u}=\left(\begin{array}{cc}
\sigma_{u 0}^{2} & \rho_{u} \sigma_{u 0} \sigma_{u 1} \\
\rho_{u} \sigma_{u 0} \sigma_{u 1} & \sigma_{u 1}^{2}
\end{array}\right)
$$

Similarly, we can define a variance-covariance matrix $\Sigma_{w}$ for items, using the standard deviations $\sigma_{w 0}, \sigma_{w 1}$, and the correlation $\rho_{w}$.

$$
\Sigma_{w}=\left(\begin{array}{cc}
\sigma_{w 0}^{2} & \rho_{w} \sigma_{w 0} \sigma_{w 1} \\
\rho_{w} \sigma_{w 0} \sigma_{w 1} & \sigma_{w 1}^{2}
\end{array}\right)
$$

The standard way to express this relationship between the subject intercepts $u_{0}$ and slopes $u_{1}$, and the item intercepts $w_{0}$ and slopes $w_{1}$, is to define a bivariate normal distribution as follows:

$$
\left(\begin{array}{l}
u_{0} \\
u_{1}
\end{array}\right) \sim \mathcal{N}\left(\left(\begin{array}{l}
0 \\
0
\end{array}\right), \Sigma_{u}\right), \quad\left(\begin{array}{l}
w_{0} \\
w_{1}
\end{array}\right) \sim \mathcal{N}\left(\left(\begin{array}{l}
0 \\
0
\end{array}\right), \Sigma_{w}\right)
$$

An important point to notice here is that any $n \times n$ variance-covariance matrix has associated with it an $n \times n$ correlation matrix. In the subject variance-covariance matrix $\Sigma_{u}$, the correlation matrix is

$$
\left(\begin{array}{cc}
1 & \rho_{01} \\
\rho_{10} & 1
\end{array}\right)
$$

In a correlation matrix, the diagonal elements will always be 1 , because a variable always has a correlation of 1 
Listing 6 - Stan code for the varying intercepts, varying slopes model.

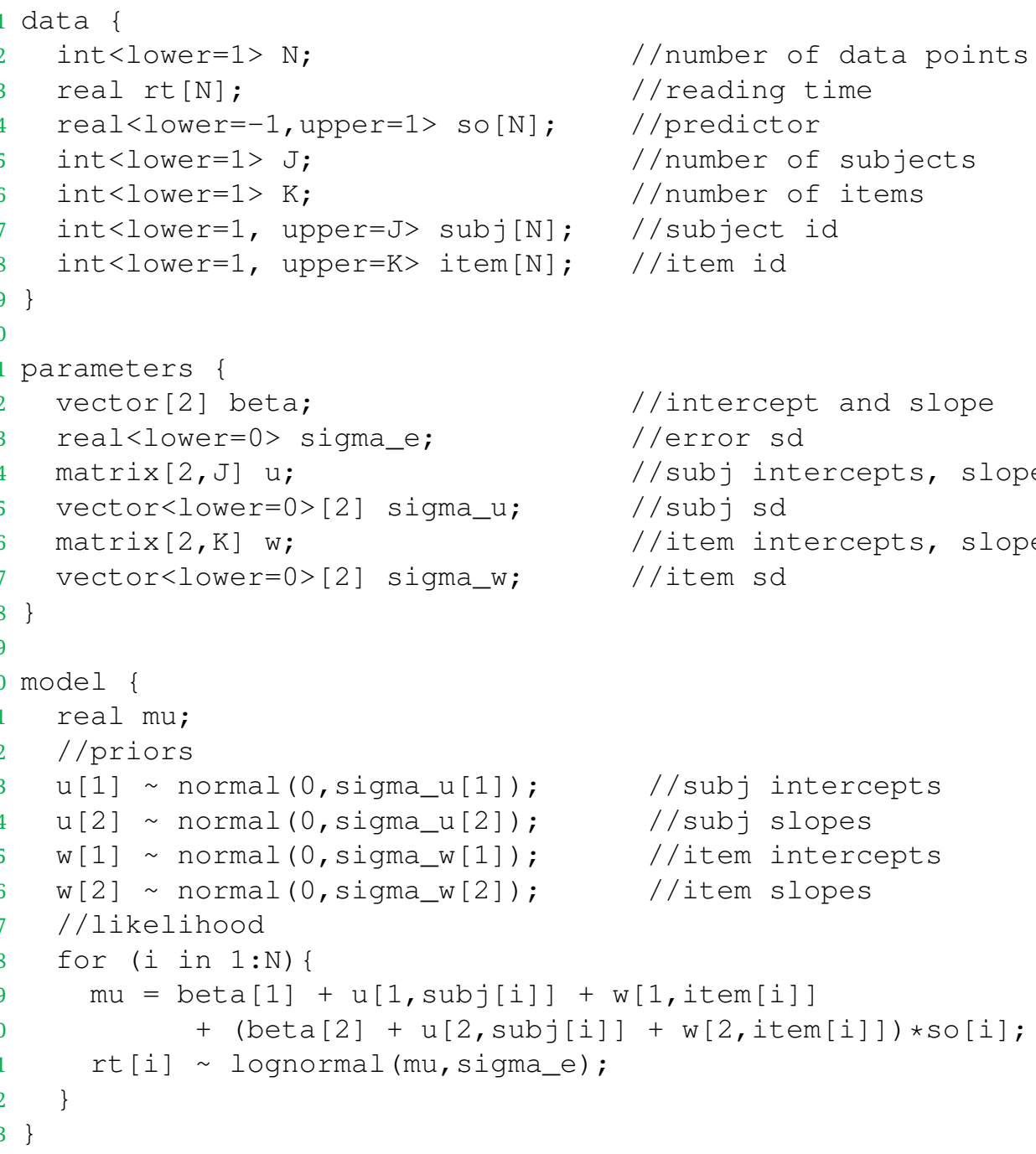

with itself. The off-diagonal entries will have the correlations between the variables. Note also that, given the variances $\sigma_{u 0}^{2}$ and $\sigma_{u 1}^{2}$, we can always recover the variancecovariance matrix, if we know the correlation matrix. This is because of the above-mentioned definition of covariance.

A correlation matrix can be factored into a matrix square root. Given a correlation matrix $C$, we can obtain its square root matrix $L$. The square root of a matrix is such that we can square $L$ to get the correlation matrix $C$ back. In the next section, we see that the matrix square root is important for generating the random intercepts and slopes because of its role in generating correlated random variables. Appendix A describes one method for obtaining $L$, namely, the Cholesky factorization.
Defining the model. With this background, implementing the varying intercepts, varying slopes model is straightforward; see Listing 7 for the R code and Listing 8 for the Stan code. The R list st anDat is identical to the one of the varying intercepts, varying slopes model, and therefore we will focus on the Stan code. The data block is the same as before. The parameters block contains several new parameters. As before, we have vectors sigma_u and sigma_w, which are $\left(\sigma_{u 0}, \sigma_{u 1}\right)^{\top}$ and $\left(\sigma_{w 0}, \sigma_{w 1}\right)^{\top}$. The variables I_u, L_w, z_u, and z_w, which have been declared in the parameters block, play a role in the transformed parameters block, a block which we did not use in the earlier models. The transformed parameters block generates the bysubject and by-item varying intercepts and slopes using the parameters sigma_u, L_u, z_u, sigma_w, L_w, and $z_{-}$w. The $J$ pairs of by-subject varying intercepts and 
Table 4 घ The quantiles and the $\hat{R}$ statistic in the Gibson and Wu data, the varying intercepts, varying slopes model.

\begin{tabular}{crrrr}
\hline parameter & mean & $2.5 \%$ & $97.5 \%$ & $\hat{R}$ \\
\hline$\hat{\beta}_{0}$ & 6.06 & 5.92 & 6.20 & 1 \\
$\hat{\beta}_{1}$ & -0.04 & -0.09 & 0.02 & 1 \\
$\hat{\sigma}_{e}$ & 0.52 & 0.48 & 0.55 & 1 \\
$\hat{\sigma}_{u 0}$ & 0.25 & 0.18 & 0.34 & 1 \\
$\hat{\sigma}_{u 1}$ & 0.06 & 0.01 & 0.13 & 1 \\
$\hat{\sigma}_{w 0}$ & 0.20 & 0.12 & 0.32 & 1 \\
$\hat{\sigma}_{w 1}$ & 0.04 & 0.01 & 0.11 & 1 \\
\hline
\end{tabular}

Listing 7 - R code for running the correlated varying intercepts, varying slopes model. Note that lines 1-10 and 14 of Listing 1 and lines $2-8$ of Listing 3 must be run first.

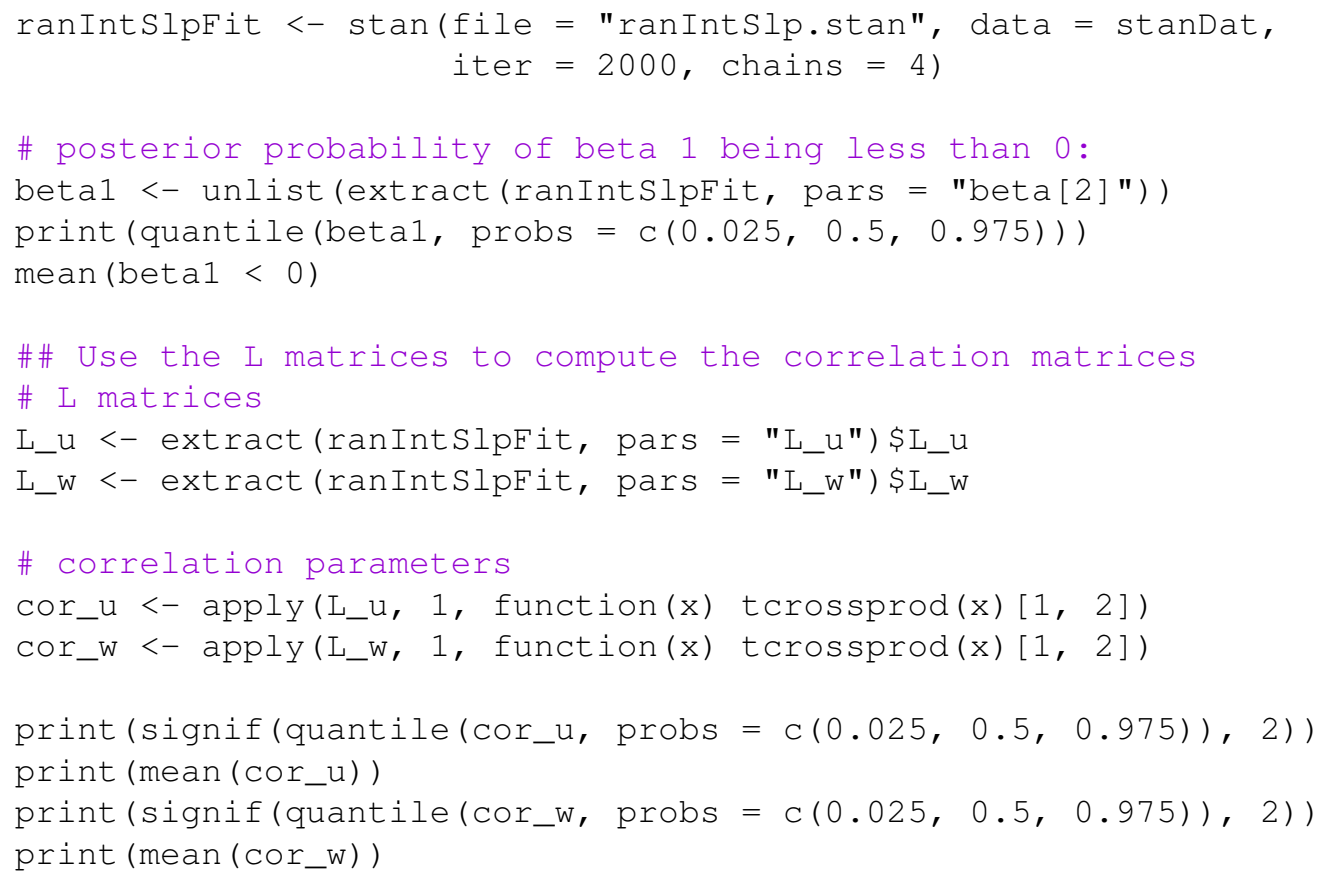

slopes are in the rows of the $J \times 2$ matrix $u$, and the $K$ pairs of by-item varying intercepts and slopes are in the rows of the $K \times 2$ matrix w.

These varying intercepts and slopes are obtained through the statements diag_pre_multiply (sigma_u, L_u) * z_u and diag_pre_multiply(sigma_w, $\left.\mathrm{L} \_w\right) * z_{\_} \mathrm{w}$. This statement generates varying intercepts and slopes from the joint probability distribution of Equation 7. The parameters L_u, L_w are the matrix square roots (Cholesky factor) of the subject and item correlation matrices, respectively, and $z_{-} u$, and $z_{-} w$ are $\mathcal{N}(0,1)$ random variables. Appendix has details on how this generates correlated random intercepts and slopes.

In the model block, we place priors on the parameters declared in the parameters block, and define how these parameters generate $\log r t$ (Listing 8, lines 30-43). The statement L_u Ikj_corr_cholesky (2.0) specifies a prior for the square root L_u (Cholesky factor) of the correlation matrix. This prior is best interpreted with respect to the square of $\mathrm{L}_{\mathrm{U}} \mathrm{u}$, that is, with respect to the correlation matrix. The statement L_u lkj_corr_cholesky (2.0) implicitly places the $\mathrm{lkj}$ prior so-called because it was first described by Lewandowski, Kurowicka, and Joe, 2009 with shape parameter $\nu=2.0$ on the correlation matrices

$$
\left(\begin{array}{cc}
1 & \rho_{u} \\
\rho_{u} & 1
\end{array}\right) \text { and }\left(\begin{array}{cc}
1 & \rho_{w} \\
\rho_{w} & 1
\end{array}\right)
$$

where $\rho_{u}$ is the correlation between the by-subject varying intercept $\sigma_{u 0}$ and slope $\sigma_{u 1}$ (cf. the covariance matrix of Equation 5) and $\rho_{w}$ is the correlation between the by-item 
varying intercept $\sigma_{w 0}$ and slope $\sigma_{w 1}$. The lkj distribution is a probability distribution over correlation matrices. The lkj distribution has one shape parameter $\nu$, which controls the prior correlation. If $\nu>1$, then the probability density becomes concentrated about the $2 \times 2$ identity matrix. ${ }^{5}$ This expresses the prior belief that the correlations are not large. If $\nu=1$, then the probability density function is uniform over all $2 \times 2$ correlation matrices. If $0<\nu<1$, then the probability density has a trough at the $2 \times 2$ identity matrix. In our model, we choose $\nu=2.0$. This choice implies that the correlations on the off-diagonal are near zero, reflecting the fact that we have no prior information about the correlation between intercepts and slopes.

The statement to_vector $\left(z_{-} u\right) \sim \operatorname{normal}(0,1)$ places a normal distribution with mean zero and standard deviation one on $z \_u .{ }^{6}$ The same goes for $z \_w$. The forloop assigns to mu the mean of the log-normal distribution from which we draw rt [i], conditional on the value of the predictor so [i] for relative clause type and the subject and item identity.

Running the model. We can now fit the varying intercepts, varying slopes model; see Listing 7 for the code. We see in the model summary in Table 5 that the model has converged, ${ }^{7}$ and that the credible intervals of the parameter of interest, $\beta_{1}$, still includes zero. In fact, the posterior probability of the parameter being less than zero is now $90 \%$. This information can be extracted as shown in Listing 7, lines 6-8.

Figure 4 plots the varying slope's posterior distribution against the varying intercept's posterior distribution for each subject. The correlation between $u_{0}$ and $u_{1}$ is negative, as captured by the marginal posterior distributions of the correlation $\rho_{u}$ between $u_{0}$ and $u_{1}$. Thus, Figure 4 suggests that the slower a subject's reading time is on average, the slower they read object relatives. In contrast, Figure 4 shows no clear pattern for the by-item varying intercepts and slopes. The broader distribution of the correlation parameter for items compared to slopes illustrates the greater uncertainty concerning the true value of the parameter. We briefly discuss inference next.

Random effects in a non-Bayesian LMM. We fit the same model also as a classical non-Bayesian LMM with the 1 mer function from the lme 4 package. This allows us to compare the 1 me 4 results with the Stan results. Here, we focus on random effects. As illustrated in Figure 5, the estimates of the random-effect standard deviations of the classical LMM are in agreement with the modes of the posterior distributions. The lmer function does not show any con- vergence error, but the correlations between the random intercepts and slopes shows the boundary values -1 and +1 ,: the variance-covariance matrices for the subject and item random effects are degenerate. By contrast, Stan can still estimate posterior distributions for parameters in such an overly complex model (Figure 4). Of course, one may want to simplify the model for reasons of parsimony, or easier interpretability. Model selection can be carried out by evaluating predictive performance of the model, with methods such as Leave One Out (LOO) Cross-validation, or by using information criteria like the Watanabe Akaike (or Widely Available) Information Criterion (WAIC). See Nicenboim and Vasishth (2016) for discussion and example code.

\section{Inference}

Having fit a correlated varying intercepts, varying slopes model, we now explain one way to carry out statistical inference, using credible intervals. We have used this approach to draw inferences from data in previously published work (e.g., Frank, Trompenaars, and Vasishth, 2015, Hofmeister and Vasishth, 2014, Safavi, Husain, and Vasishth, 2016). There are of course other approaches possible for carrying out inference. Bayes Factors are an example; see Lee and Wagenmakers (2013) and Rouder and Morey (2012). Another is to define a Region of Practical Equivalence (Kruschke, 2015). The reader can choose the approach they find the most appealing. For further discussion of Bayes Factors, with example code, see Nicenboim and Vasishth (2016).

The result of fitting the varying intercepts, varying slopes model is the posterior distribution of the model parameters. Direct inference from the posterior distributions is possible. For instance, we can find the posterior probability with which the fixed intercept $\beta_{1}$ or the correlation $\rho_{u}$ between by-subject varying intercepts and slopes take on any given value by consulting the marginal posterior distributions whose histograms are shown in Figure 6. The information conveyed by such graphs can be sharpened by using the $95 \%$ credible interval, mentioned earlier. Approximately $95 \%$ of the posterior density of $\beta_{1}$ lies between the 2.5 th percentile -0.09 and the 97.5 th percentile 0.02 . This leads us to conclude that the slope $\beta_{1}$ for relative clause type so is less than zero with probability $90 \%$ (see Listing 7, line 8). Since zero is included in the credible interval, it is difficult to draw the inference that object relative clauses are read faster than subject relative clauses. However, one could perhaps still make a weak claim that

\footnotetext{
${ }^{5}$ The lkj prior can scale up to correlation matrices larger than $2 \times 2$.

${ }^{6}$ The function to_vector means that we rearrange the matrix $z$ _u as a vector in order to place the normal distribution on a vector. This makes the code run faster.

${ }^{7}$ We do not report the R-hat statistic for parameters $\rho_{u}, \rho_{w}$ because these parameters converge when $\hat{R}$ equals one for each entry of the matrices $L_{u}, L_{w}$. This was the case.
} 
Table 5 - The quantiles and the $\hat{R}$ statistic in the Gibson and Wu data, the varying intercepts, varying slopes model.

\begin{tabular}{crrrr}
\hline parameter & mean & $2.5 \%$ & $97.5 \%$ & $\hat{R}$ \\
\hline$\hat{\beta}_{0}$ & 6.06 & 5.92 & 6.20 & 1 \\
$\hat{\beta}_{1}$ & -0.04 & -0.09 & 0.02 & 1 \\
$\hat{\sigma}_{e}$ & 0.52 & 0.48 & 0.55 & 1 \\
$\hat{\sigma}_{u 0}$ & 0.25 & 0.18 & 0.34 & 1 \\
$\hat{\sigma}_{u 1}$ & 0.07 & 0.01 & 0.13 & 1 \\
$\hat{\sigma}_{w 0}$ & 0.20 & 0.12 & 0.32 & 1 \\
$\hat{\sigma}_{w 1}$ & 0.04 & 0.0 & 0.11 & 1 \\
$\hat{\rho}_{u}$ & -0.44 & -0.91 & 0.36 & \\
$\hat{\rho}_{w}$ & -0.01 & -0.76 & 0.76 & \\
\hline
\end{tabular}

Figure 4 ॥ The top row shows the relationship between the posterior mean of the varying slopes (y-axis) and intercepts (x-axis) for each subject (left panel) and item (right panel). The bottom row shows the posterior distribution of the parameter of correlation between the varying slopes and intercepts for each subject (left panel) and item (right panel).
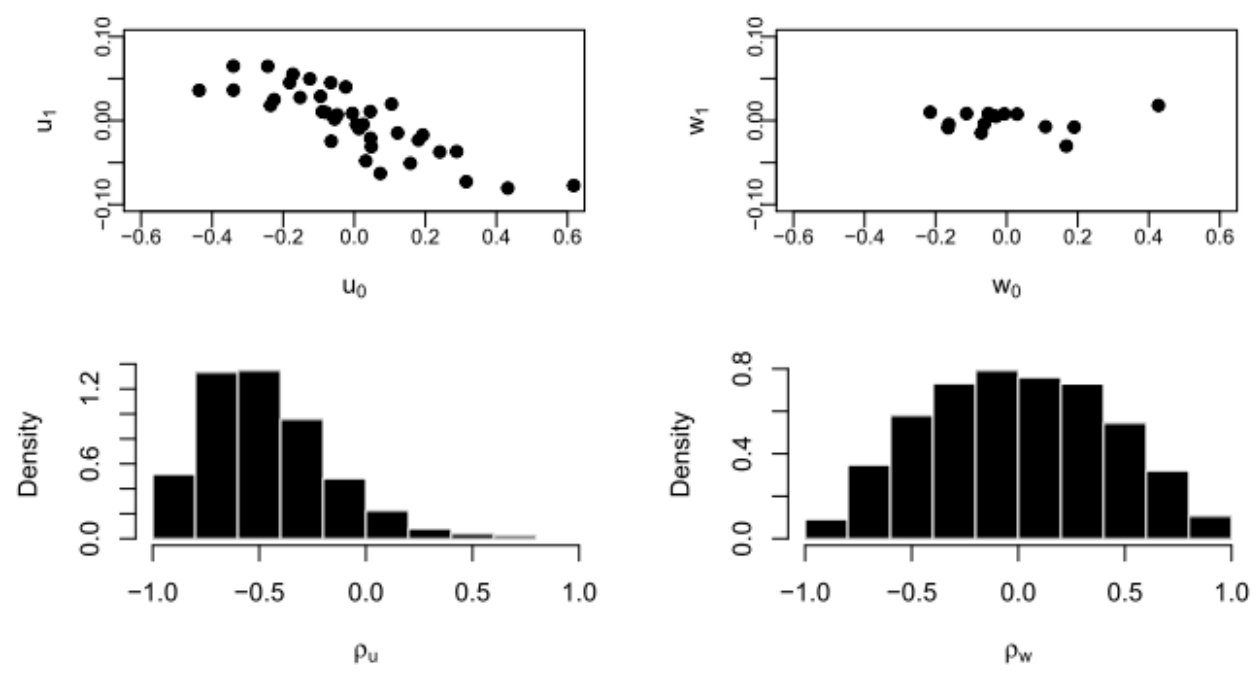

object relatives are easier to process, especially if a lot of evidence has accumulated in other experiments that supports such a conclusion (see Vasishth, Chen, Li, and Guo, 2013 for a more detailed discussion). Meta-analysis of existing studies can help in obtaining a better estimate of the posterior distribution of a parameter; for psycholinguistic examples, see Vasishth (2015), Jäger, Engelmann, and Vasishth (2016), Mahowald, James, Futrell, and Gibson (2016), Vasishth et al. (2013).

What about the correlations between varying intercepts and varying slopes for subject and for item? What can we infer from the analysis about these relationships? The $95 \%$ credible interval for $\rho_{u}$ is $(-1,0.1)$. Our belief that $\rho_{u}$ is less than zero is rather uncertain, although we can conclude that $\rho_{u}$ is less than zero with probabil-

ity $90 \%$. There is only weak evidence that subjects who read faster than average exhibit greater slowdowns at the head noun of object relative clauses than subjects who read slower than average. For the by-item varying intercepts and slopes, it is pretty clear that we do not have enough data (15 items) to draw any conclusions. For these data, it probably makes sense to fit a simpler model (Bates, Kliegl, et al., 2015), with only varying intercepts and slopes for subject, and only varying intercepts for items; although there is no harm done in this particular example if we fit a model with a full variance-covariance matrix for both subjects and items.

In sum, regarding our main research question, our conclusion here is that we cannot say that object relatives are harder to process than subject relatives, because the cred- 
Figure 5 - The curves show the density of the posterior distributions of the random-effect standard deviations. The vertical bars indicate the corresponding lmer estimates. The top row shows the random effects for subjects, the bottom row shows the random effects for items. Left-hand panels correspond to random intercepts, right-hand panels correspond to random slopes.
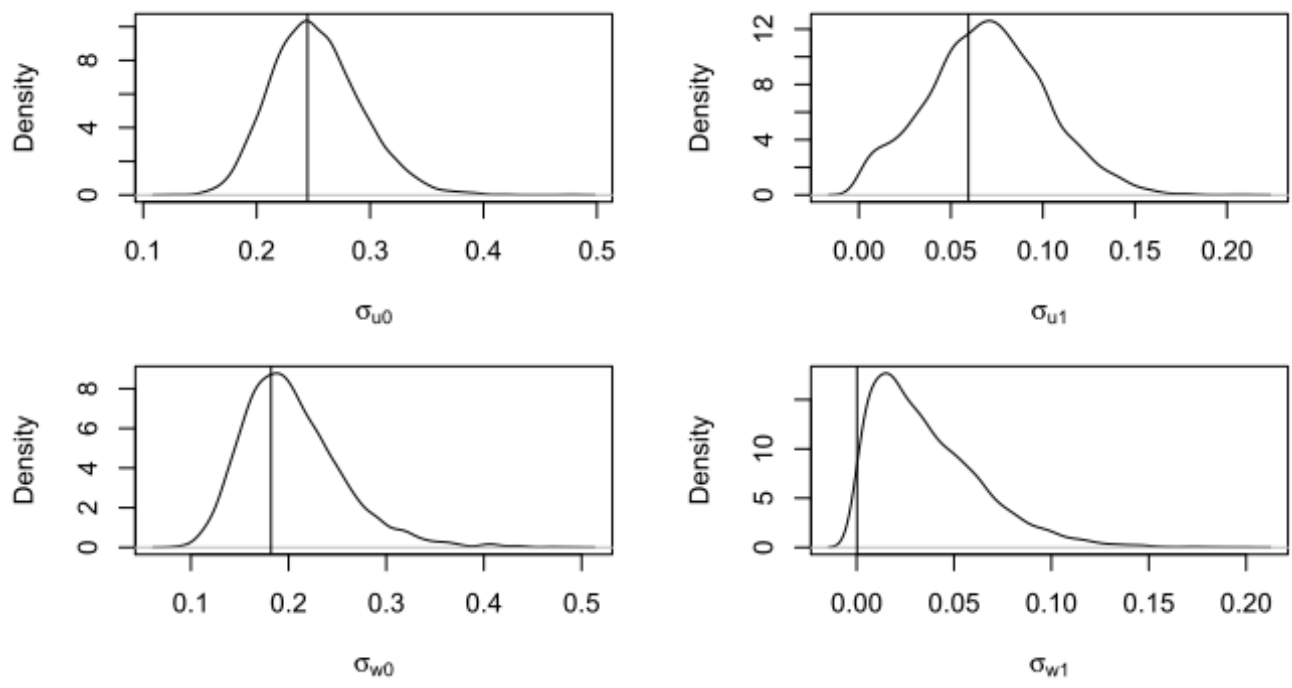

ible interval for $\beta_{1}$ includes zero. However, one could argue that there is some weak evidence in favor of the hypothesis, since the posterior probability of the parameter being negative is approximately $90 \%$.

\section{Further reading}

We hope that this tutorial has given the reader a flavor of what it would be like to fit Bayesian linear mixed models. There is of course much more to say on the topic, and we hope that the interested reader will take a look at some of the excellent books that have recently come out. We suggest below a sequence of reading that we found helpful. A good first general textbook is by Gelman and Hill (2007); it begins with the frequentist approach and only later transitions to Bayesian models. The book by McElreath (2016) is also excellent. For those looking for a psychology-specific introduction, the books by Kruschke (2015) and Lee and Wagenmakers (2013) are to be recommended, although for the latter the going might be easier if the reader has already looked at Gelman and Hill (2007). As a second book, Lunn et al. (2012) is recommended; it provides many interesting and useful examples using the BUGS language, which are discussed in exceptionally clear language. Many of these books use the BUGS syntax (Lunn et al., 2000), which the probabilistic programming language JAGS (Plummer, 2012) also adopts; however, Stan code for these books is slowly becoming avail-

able on the Stan home page (https://github.com/stan-dev/ example-models/wiki). For those with introductory calculus, a slightly more technical introduction to Bayesian methods by Lynch (2007) is an excellent choice. Finally, the textbook by Gelman et al. (2013) is the definitive modern guide, and provides a more advanced treatment.

\section{Authors' note}

We are grateful to the developers of Stan (in particular, Andrew Gelman, Bob Carpenter) and members of the Stan mailing list for their advice regarding model specification. Douglas Bates and Reinhold Kliegl have helped considerably over the years in improving our understanding of LMMs from a frequentist perspective. We also thank Edward Gibson for releasing his published data. Titus von der Malsburg, Lena Jäger, and Bruno Nicenboim provided useful comments on previous drafts. Thanks also go to Charles S. Stanton for catching a mistake in our code.

\section{References}

Baayen, R. H., Bates, D. M., Kliegl, R., \& Vasishth, S. (2015). RePsychLing: Data sets from psychology and linguistics experiments. R package version 0.0.4. Retrieved from https://github.com/dmbates/RePsychLing

Baayen, R. H., Vasishth, S., Bates, D. M., \& Kliegl, R. (2016). The cave of shadows: Addressing the human 
Figure 6 - Upper and lower bounds on the highest posterior density credible intervals (dashed lines) plotted over the marginal posterior distribution of the fixed slope $\beta_{1}$ (left) and of the correlation $\rho_{u}$ between the by-subject varying intercepts and varying slopes (right).

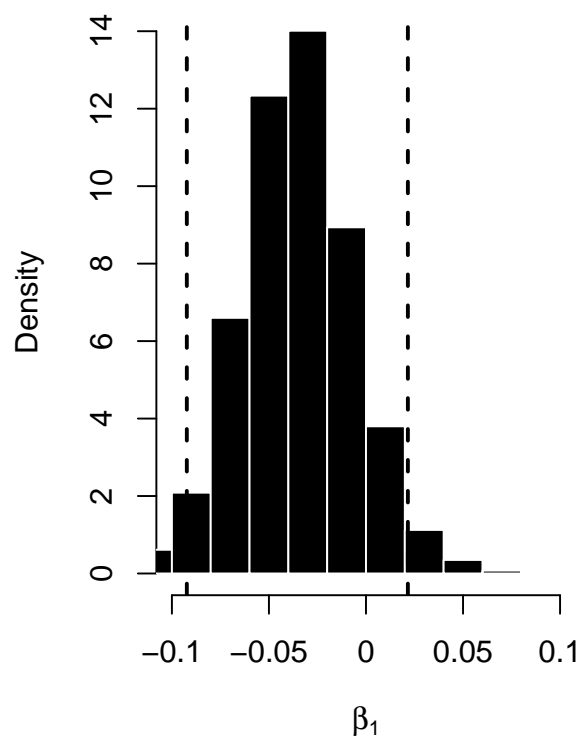

factor with generalized additive mixed models. arXiv preprint, arXiv:1511.03120.

Barr, D. J., Levy, R., Scheepers, C., \& Tily, H. J. (2013). Random effects structure for confirmatory hypothesis testing: Keep it maximal. Journal of Memory and Language, 68(3), 255-278. doi:10.1016/j.jml.2012.11.001

Bates, D. M., Kliegl, R., Vasishth, S., \& Baayen, H. (2015). Parsimonious mixed models. ArXiv e-print arXiv:1506.04967; submitted to Journal of Memory and Language. Retrieved from http://arxiv.org/abs/ 1506.04967

Bates, D. M., Mächler, M., Bolker, B. M., \& Walker, S. C. (2015). Fitting linear mixed-effects models using lme4. Journal of Statistical Software, 67(1), 1-48. doi:10.18637/jss.v067.i01. eprint: 1406.5823

Frank, S. L., Trompenaars, T., \& Vasishth, S. (2015). Crosslinguistic differences in processing double-embedded relative clauses: Working-memory constraints or language statistics? Cognitive Science, 554-578. doi:10 . 1111/cogs.12247

Gelman, A. (2006). Prior distributions for variance parameters in hierarchical models (comment on article by Browne and Draper). Bayesian Analysis, 1(3), 515-534.

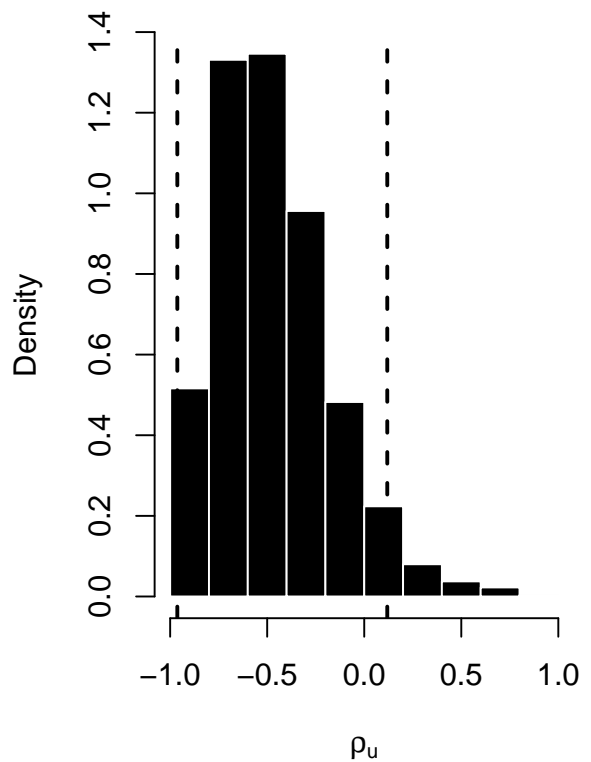

Gelman, A., Carlin, J. B., Stern, H. S., Dunson, D. B., Vehtari, A., \& Rubin, D. B. (2013). Bayesian data analysis (3rd edition). Boca Raton, FL, USA: Chapman and Hall/CRC.

Gelman, A. \& Hill, J. (2007). Data analysis using regression and multilevel/hierarchical models. Cambridge, UK: Cambridge University Press.

Gelman, A. \& Rubin, D. B. (1992). Inference from iterative simulation using multiple sequences. Statistical Science, 457-472.

Gibson, E. \& Wu, H.-H. I. (2013). Processing Chinese relative clauses in context. Language and Cognitive Processes, 28(1-2), 125-155. doi:0.1080/01690965.2010.536656

Hofmeister, P. \& Vasishth, S. (2014). Distinctiveness and encoding effects in online sentence comprehension. Frontiers in Psychology, 5, 1237. doi:10.3389/fpsyg. 2014.01237

Hsiao, F. P.-F. \& Gibson, E. (2003). Processing relative clauses in Chinese. Cognition, 90, 3-27. doi:10.1016/ S0010-0277(03)00124-0

Jäger, L. A., Engelmann, F., \& Vasishth, S. (2016). Similaritybased interference in sentence comprehension: literature review and bayesian meta-analysis. Manuscript submitted. 
Just, M. \& Carpenter, P. (1992). A capacity theory of comprehension: Individual differences in working memory. Psychological Review, 99(1), 122-149. doi:10.1037/ 0033-295X.99.1.122

Kliegl, R., Wei, P., Dambacher, M., Yan, M., \& Zhou, X. (2010). Experimental effects and individual differences in linear mixed models: Estimating the relationship between spatial, object, and attraction effects in visual attention. Frontiers in Psychology, 1, 238. doi:10.3389/ fpsyg.2010.00238

Kruschke, J. (2015). Doing Bayesian Data Analysis: A tutorial with $R$, JAGS, and Stan. London, UK: Academic Press.

Lavine, M. (1999). What is Bayesian statistics and why everything else is wrong. The Journal of Undergraduate Mathematics and Its Applications, 20, 165-174. Retrieved from https://www2. stat.duke.edu/courses / Spring06/sta114/whatisbayes.pdf

Lee, M. D. \& Wagenmakers, E.-J. (2013). Bayesian cognitive modeling: A practical course. Cambridge, UK: Cambridge University Press.

Lewandowski, D., Kurowicka, D., \& Joe, H. (2009). Generating random correlation matrices based on vines and extended onion method. Journal of Multivariate Analysis, 100(9), 1989-2001.

Lunn, D. J., Jackson, C., Spiegelhalter, D. J., Best, N., \& Thomas, A. (2012). The BUGS book: A practical introduction to Bayesian analysis. Boca Raton, FL, USA: CRC Press.

Lunn, D. J., Thomas, A., Best, N., \& Spiegelhalter, D. (2000). WinBUGS - A Bayesian modelling framework: Concepts, structure, and extensibility. Statistics and Computing, 10(4), 325-337. doi:10.1023/A:1008929526011

Lynch, S. M. (2007). Introduction to applied Bayesian statistics and estimation for social scientists. New York, NY, USA: Springer. doi:10.1007/978-0-387-71265-9

Mahowald, K., James, A., Futrell, R., \& Gibson, E. (2016). A meta-analysis of syntactic priming in language production. Journal of Memory and Language.

Matuschek, H., Kliegl, R., Vasishth, S., Baayen, R. H., \& Bates, D. M. (2016). Balancing Type I Error and Power in Linear Mixed Models. arXiv preprint arXiv:1511.01864.

McElreath, R. (2016). Statistical rethinking: A Bayesian course with examples in $R$ and Stan. Texts in Statistical Science. Boca Raton, FL, USA: Chapman and Hall/CRC.

Morey, R. D., Hoekstra, R., Rouder, J. N., Lee, M. D., \& Wagenmakers, E.-J. (2015). The fallacy of placing confidence in confidence intervals. Psychonomic Bulletin \& Review, 23(1), 103-123. doi:10.3758/s13423-015-09478
Nicenboim, B. \& Vasishth, S. (2016). Statistical methods for linguistics research: Foundational ideas - Part II. arXiv preprint arXiv:1602.00245.

Pinheiro, J. C. \& Bates, D. M. (2000). Mixed-effects models in $S$ and S-PLUS. New York: Springer. doi:10.1007/b98882

Plummer, M. (2012). JAGS version 3.3.0 manual. International Agency for Research on Cancer. Lyon, France.

R Development Core Team. (2006). $R$ : A language and environment for statistical computing. ISBN 3-900051-07-0. R Foundation for Statistical Computing. Vienna, Austria. Retrieved from http://www.R-project.org

Raftery, A. E. \& Lewis, S. (1992). How many iterations in the Gibbs sampler? In J. Bernardo, J. Berger, A. Dawid, \& A. Smith (Eds.), Bayesian statistics 4 (pp. 763-773). Oxford, UK: Oxford University Press.

Rouder, J. N. (2005). Are unshifted distributional models appropriate for response time? Psychometrika, 70, 377-381. doi:10.1007/s11336-005-1297-7

Rouder, J. N. \& Morey, R. D. (2012). Default Bayes factors for model selection in regression. Multivariate Behavioral Research, 47(6), 877-903. doi:10.1080/00273171. 2012.734737

Safavi, M. S., Husain, S., \& Vasishth, S. (2016). Dependency resolution difficulty increases with distance in persian separable complex predicates: Implications for expectation and memory-based accounts. Frontiers in Psychology, 7. Special Issue on Encoding and Navigating Linguistic Representations in Memory. doi:10. 3389/fpsyg.2016.00403

Spiegelhalter, D. J., Abrams, K. R., \& Myles, J. P. (2004). Bayesian approaches to clinical trials and health-care evaluation. Chichester, UK: John Wiley \& Sons. doi:10. 1002/0470092602

Stan Development Team. (2014). Stan modeling language users guide and reference manual, version 2.4. Retrieved from http://mc-stan.org/

Vasishth, S. (2015). A meta-analysis of relative clause processing in Mandarin Chinese using bias modelling. Sheffield, UK. Retrieved from http://www.ling. unipotsdam.de/ vasishth/pdfs/VasishthMScStatistics.pdf

Vasishth, S., Chen, Z., Li, Q., \& Guo, G. (2013). Processing Chinese relative clauses: Evidence for the subjectrelative advantage. PLoS ONE, 8(10), e77006. doi:10. 1371/journal.pone.0077006

Vasishth, S. \& Nicenboim, B. (2016). Statistical methods for linguistic research: Foundational ideas - Part I. Language and Linguistics Compass, 10, 349-369. doi:10 . 1111/lnc3.12201

Xie, Y. (2015). knitr: A general-purpose package for dynamic report generation in $R$. R package version 1.11 . 
Figure 7 - Uncorrelated random variables $\mathbf{z}=\left(z_{1}, z_{2}\right)^{\boldsymbol{\top}}$ (panel a) and correlated random variables $\mathbf{x}=\left(x_{1}, x_{2}\right)^{\boldsymbol{\top}}($ panel b).

(a)

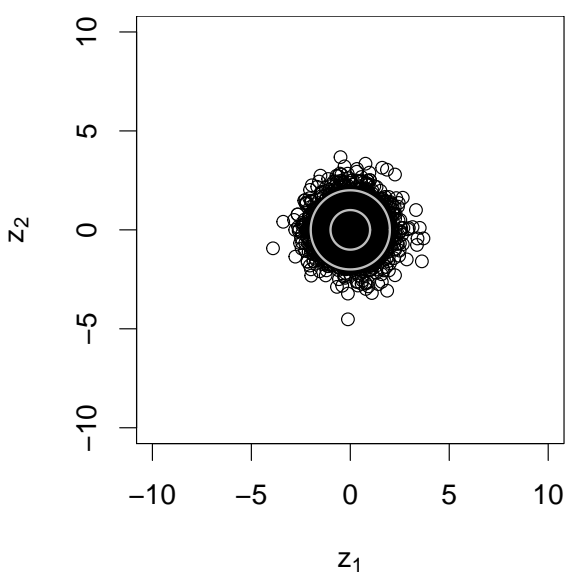

(b)

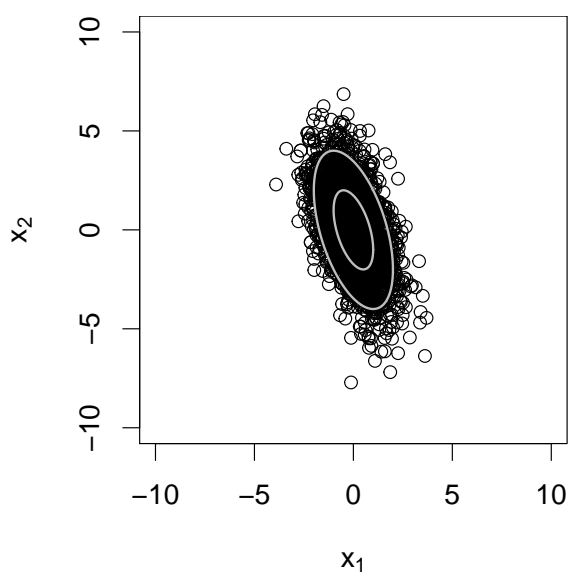

\section{Appendix A: Cholesky factorization}

A correlation matrix can be factored into a square root of the matrix; one method is the Cholesky factorization. Given a correlation matrix $C$, we can obtain its square root $L$. The square root of a matrix is such that we can square $L$ to get the correlation matrix $C$ back. We illustrate the matrix square root with a simple example. Suppose we have a correlation matrix:

$$
C=\left(\begin{array}{cc}
1 & -0.5 \\
-0.5 & 1
\end{array}\right)
$$

We can use the Cholesky factorization function in R, chol, to derive the lower triangular square root $L$ of this matrix. This gives us:

$$
L=\left(\begin{array}{cc}
1 & 0 \\
-0.5 & 0.8660254
\end{array}\right)
$$

We confirm that this is a square root by multiplying $L$ with itself to get the correlation matrix back (squaring a matrix is done by multiplying the matrix by its transpose):

$$
L L^{\top}=\left(\begin{array}{cc}
1 & 0 \\
-0.5 & 0.8660254
\end{array}\right)\left(\begin{array}{cc}
1 & -0.5 \\
0 & 0.8660254
\end{array}\right)=\left(\begin{array}{cc}
1 & -0.5 \\
-0.5 & 1
\end{array}\right)
$$

The reason that the Cholesky factorization is useful for LMMs is that we use it to generate the by-subject and by-item random intercepts and slopes.

Generating correlated random variables using the Cholesky factor. The by-subject and by-item adjustments are generated using the following standard procedure for generating correlated random variables $\mathbf{x}=\left(x_{1}, x_{2}\right)$ :

1. Given a vector of standard deviations (e.g., $\sigma_{u 0}, \sigma_{u 1}$ ), create a diagonal matrix:

$$
\tau=\left(\begin{array}{cc}
\sigma_{u 0} & 0 \\
0 & \sigma_{u 0}
\end{array}\right)
$$

2. Premultiply the diagonalized matrix $\tau$ with the Cholesky factor $L$ of the correlation matrix $C$ to get a matrix $\Lambda$.

3. Generate values from a random variable $\mathbf{z}=\left(z_{1}, z_{2}\right)^{\top}$, where $z_{1}$ and $z_{2}$ each have independent $\mathcal{N}(0,1)$ distributions (left panel of Figure 7).

4. Multiply $\Lambda$ with $\mathbf{z}$; this generates the correlated random variables $\mathbf{x}$ (right panel of Figure 7).

It is helpful to walk through steps 1 to 4 of the procedure described above for generating correlated random intercepts and random slopes. These are carried out in lines 26 and 36 of Listing 8. The statement diag_pre_multiply (sigma_u, 
L_u ) in line 26 computes the transpose matrix product (steps 1 and 2). The statement to_vector (z_u) normal ( 0 , 1 ) ; in line 36 generates z_u as samples from the unit normal distribution (step 3). In line 26, the right multiplication of diag_pre_multiply (sigma_u, L_u) by z_u, a matrix of normally distributed random variables, yields the varying intercepts and slopes (step 4).

$$
\begin{aligned}
\left(\begin{array}{cc}
u_{01} & u_{11} \\
u_{02} & u_{12} \\
\vdots & \vdots \\
u_{0 J} & u_{1 J}
\end{array}\right) & =\left(\operatorname{diag}\left(\sigma_{u 0}, \sigma_{u 1}\right) L_{u} \mathbf{z}_{u}\right)^{\top} \\
& =\left(\left(\begin{array}{cc}
\sigma_{u 0} & 0 \\
0 & \sigma_{01}
\end{array}\right)\left(\begin{array}{cc}
\ell_{11} & 0 \\
\ell_{21} & \ell_{22}
\end{array}\right)\left(\begin{array}{llll}
z_{11} & z_{12} & \ldots & z_{1 J} \\
z_{21} & z_{22} & \ldots & z_{2 J}
\end{array}\right)\right)^{\top}
\end{aligned}
$$

\section{Appendix B: Matrix formulation of the linear mixed model}

In the body of the text, we fit four models of increasing complexity to the data-set of Gibson and Wu (2013). In all specifications, there was an explicit vector so for the predictor variable in Stan. However, if we want to fit more complex models with many categorical and continuous predictors and interactions, this approach requires increasingly complex specifications in Stan code. Alternatively, we can use the matrix formulation of the linear mixed model that allows for using the same code for models of different complexity. In the following, we will apply this approach for an alternative version of the correlated varying intercepts, varying slopes model, which includes random intercepts and slopes for subjects and items.

We build up the model specification by first noting that, for each subject, the by-subject varying intercept $u_{0}$ and slope $u_{1}$ have a multivariate normal prior distribution with mean zero and covariance matrix $\Sigma_{u}$. Similarly, for each item, the by-item varying intercept $w_{0}$ and slope $w_{1}$ have a multivariate normal prior distribution with mean zero and covariance matrix $\Sigma_{w}$. The error $\varepsilon$ is assumed to have a normal distribution with mean zero and standard deviation $\sigma_{e}$.

We proceed to implement the model in Stan. Instead of passing the predictor so to stan as vector, as we did earlier, we make so into a design matrix X using the function model . matrix available in $\mathrm{R}$ (see Listing 9 , line 2 ). ${ }^{8}$ The command model matrix ( 1 + so, rDat) creates a model matrix with two fixed effects, the intercept (1) and a factor (so), based on the data frame rDat. The first column of the design matrix X consists of all ones; this column represents the intercept. The second column is the predictor so and consists of values in $\{-1,1\}$. The model matrix thus consists of a two-level factorial design, with blocks of this design repeated for each subject. For the full data-set, we could write it very compactly in matrix form as follows:

$$
\log r t=\mathbf{X} \boldsymbol{\beta}+\mathbf{Z}_{u} \mathbf{u}+\mathbf{Z}_{w} \mathbf{w}+\varepsilon
$$

Here, $\mathbf{X}$ is the $N \times P$ model matrix (with $N=547$, since we have 547 data points; and $P=2$ since we have the intercept plus another fixed effect), $\boldsymbol{\beta}$ is a vector of length $P$ including fixed effects parameters, $\mathbf{Z}_{u}$ and $\mathbf{Z}_{w}$ are the subject and item model matrices $(N \times P)$, and $\mathbf{u}$ and $\mathbf{w}$ are the by-subject and by-item adjustments to the fixed effects estimates; these are identical to the design matrix $\mathbf{X}$ in the model with varying intercepts and varying slopes included. For more examples of similar model specifications in Stan, see the R package ReP sychLing on github (https: //github.com/dmbates/RePsychLing).

Note that we remove the column names and the attributes of the model matrix X in order to use it for Stan; refer to Listing 9. Having defined the model, we proceed to assemble the list stanDat of data, relying on the above matrix formulation. The number $\mathrm{N}$ of observations, the number $\mathrm{J}$ of subjects and $\mathrm{K}$ of items, the reading times $r t$, and the subject and item indicator variables sub $j$ and $i$ tem are familiar from the previous models presented. The integer $P$ is the number of fixed effects (two including the intercept). Model 4 includes a varying intercept $u_{0}$ and a varying slope $u_{1}$ for each subject, and so the number $\mathrm{n} \_u$ of by-subject random effects equals P. Likewise, Model 4 includes a varying intercept $w_{0}$ and a varying slope $w_{1}$ for each item, and so the number $n \_w$ of by-item random effects also equals $\mathrm{P}$.

We also have to adapt the Stan code to the model formulation (see Listing 10). The data block contains the corresponding variables. Using the command row_vector $[\mathrm{P}] \mathrm{X}[\mathrm{N}]$, we declare the fixed effects design matrix $\mathrm{X}$ as an array of $\mathrm{N}$ row vectors of length $\mathrm{P}$ whose components are the predictors associated with the $\mathrm{N}$ reading times. Likewise

\footnotetext{
${ }^{8} \mathrm{Here}$, we would like to acknowledge the contribution of Douglas Bates in specifying the model in this general matrix form.
} 
for the subject and item random effects design matrices $Z_{-} u_{u}$ and $Z_{\_}$w, which correspond to $\mathbf{Z}_{u}$ and $\mathbf{Z}_{w}$ respectively in Equation 15. The vector bet a contains the fixed effects $\beta_{0}$ and $\beta_{1}$. The matrices $L_{-} u, L_{-} w$ and the arrays $z \_u$, z_w of vectors (not to be confused with the design matrices z_u and z_w) will generate the varying intercepts and slopes $u_{0}, u_{1}$ and $w_{0}, w_{1}$, using the procedure described for the varying intercepts, varying slopes model. For example, the command vector $\left[n \_u\right] \quad u[J]$ specifies $u$ as an array of $J$ vectors of length $n \_u$; hence, there is one vector per subject. The vector sigma_u contains the standard deviations of the by-subject varying intercepts and slopes $u_{0}, u_{1}$, and the vector sigma_w contains the standard deviations of the by-item varying intercepts and slopes $w_{0}, w_{1}$. The variable sigma_e is the standard deviation $\sigma_{e}$ of the error $\varepsilon$. The transformed parameters block generates the by-subject intercepts and slopes $u_{0}, u_{1}$ and the by-item intercepts and slopes $w_{0}, w_{1}$.

We place lkj priors on the random effects correlation matrices through the $1 \mathrm{kj}$ _corr_cholesky (2.0) priors on their Cholesky factors $L_{-} u$ and $L_{-}$w. We implicitly place uniform priors on the fixed effects $\beta_{0}, \beta_{1}$, the random effects standard deviations $\sigma_{u 0}, \sigma_{u 1}$, and $\sigma_{w 0}, \sigma_{w 1}$ and the error standard deviation $\sigma_{e}$ by omitting any prior specifications for them in the model block. We specify the likelihood with the probability statement that $r t[i]$ is distributed lognormally with mean X[i] * beta + z_u[i] * u[subj[i] + z_w[i] *w[item[i]] and standard deviation sigma_e. The next step towards model-fitting is to pass the list stanDat to stan, which compiles a C++ program to sample from the posterior distribution of the model parameters.

A major advantage of the above matrix formulation is that we do not need to write a new Stan model for a future repeated measures design. All we have to do now is define the design matrix $\mathbf{X}$ appropriately, and include it (along with appropriately defined $\mathbf{Z}_{u}$ and $\mathbf{Z}_{w}$ for the subjects and items random effects) as part of the data specification that is passed to Stan.

\section{Citation}

Sorensen, T., Hohenstein, S., \& Vasishth, S. (2016). Bayesian linear mixed models using Stan: A tutorial for psychologists, linguists, and cognitive scientists. The Quantitative Methods for Psychology, 12(3), 175-200. doi:10.20982/tqmp.12.3. p175

Copyright $\odot$ 2016, Sorensen, Hohenstein, Vasishth . This is an open-access article distributed under the terms of the Creative Commons Attribution License (CC BY). The use, distribution or reproduction in other forums is permitted, provided the original author(s) or licensor are credited and that the original publication in this journal is cited, in accordance with accepted academic practice. No use, distribution or reproduction is permitted which does not comply with these terms.

Received: 28/06/2016 Accepted: 13/07/2016

Listings 8 to 10 follows. 
Listing 8 - The Stan code for the correlated varying intercepts, varying slopes model.

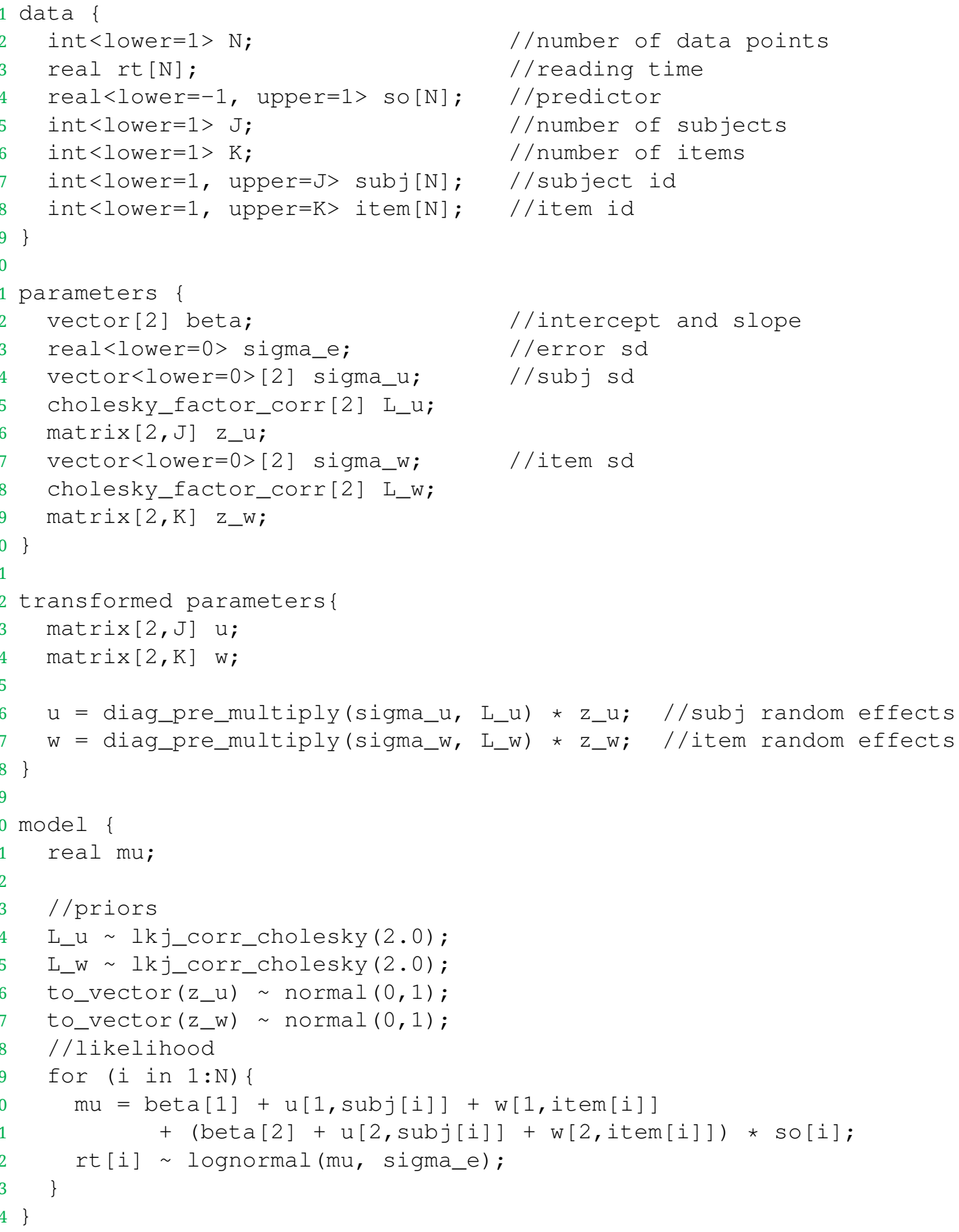


Listing 9 a Matrix formulation code for running the varying intercepts, varying slopes model.

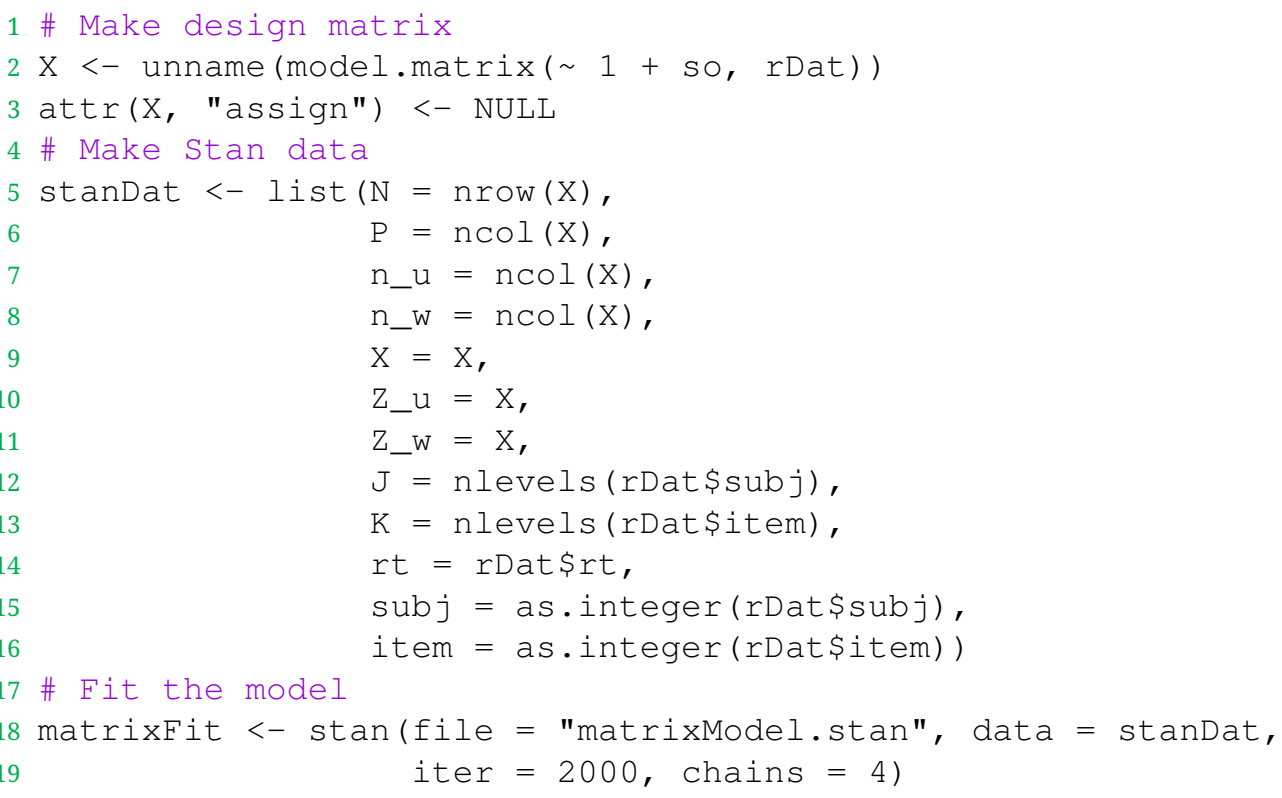


Listing 10 - Stan code for the matrix formulation of the varying intercepts, varying slopes model.

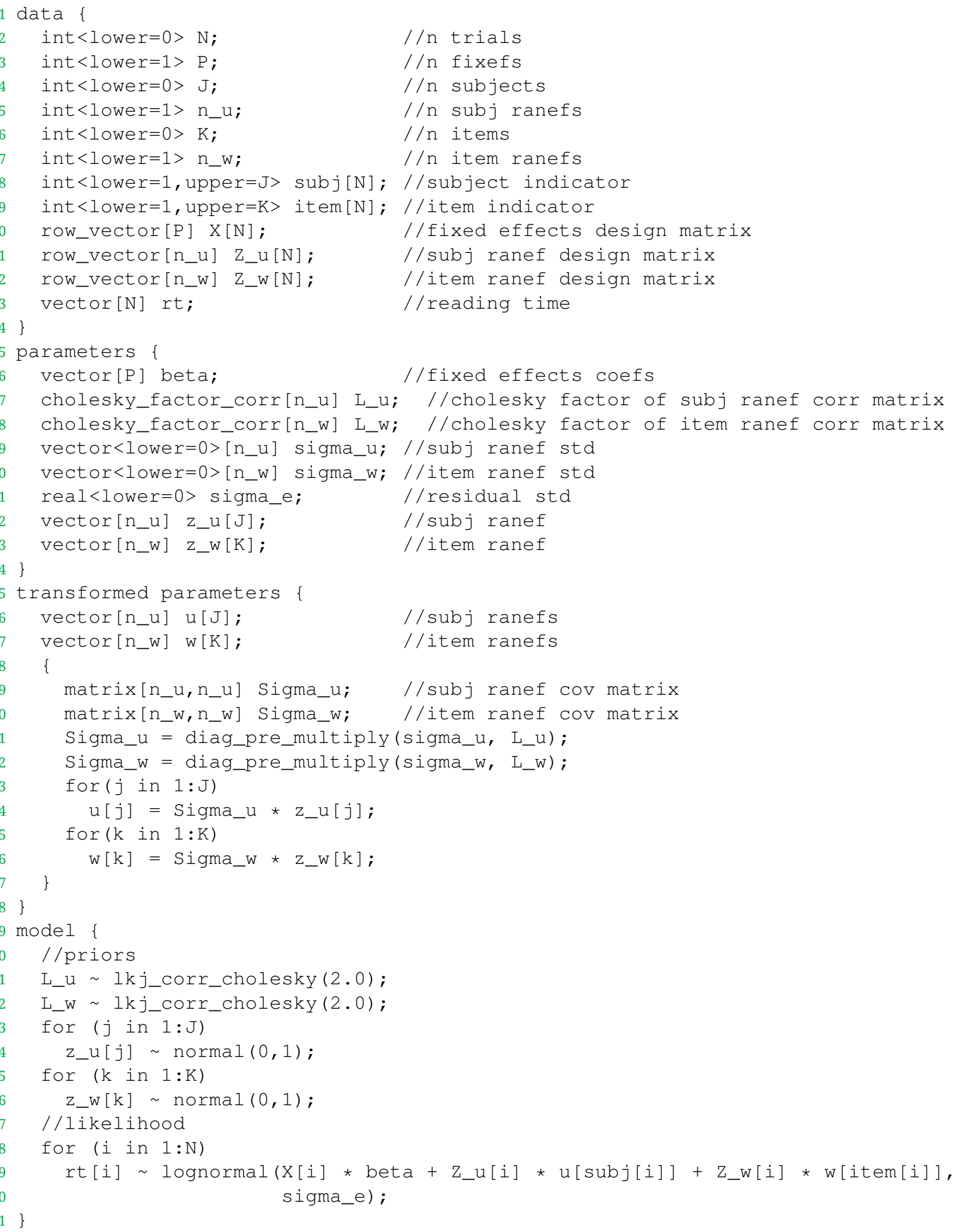

